\title{
Zypern in der Spätbronzezeit: Ein „kulturelles Konglomerat“?
}

\begin{abstract}
Prehistoric Cyprus is characterised by several scholars as a so-called cultural hybrid, a 'cultural conglomerate' so to speak and indicators of this process are said to have manifest themselves in material culture throughout the Late Bronze and Early Iron Age.

This article deals with this conceptualization of a hybrid culture, and the role of material culture and languages in its definition. If we understand Cyprus during Late Bronze Age as a cultural conglomerate or some form of cultural hybrid, is it in fact fundamentally different in concept from the old idea of a "people"? How can we speak of an unified culture, when at the same time the development of this culture should have taken place from a variety of external influences?

I argue that not only the idea of a „people“ has been preserved this way but also that cultural-historical explanatory patterns are still unconsciously derived from this principle. With a prevailing expectation to be able to see thess hybrid arrangements in the archaeological material, the concept of a Cypriot "people" is created through the metaphorical back door.
\end{abstract}

\section{1 „Sprache“ und „Volk“ in der prähistorischen Archäologie}

Die akademische Disziplin der Ur- und Frühgeschichte (oder Vor- und Frühgeschichte) wird als eine historische Kulturwissenschaft verstanden, die sich den Abschnitten der Menschheitsgeschichte widmet, für die (fast) keine eigenen schriftlichen Überlieferungen vorliegen. Die Bezeichnung „Volk“ oder „Stamm“

\footnotetext{
Anmerkung: An dieser Stelle möchte ich mich für die Einladung zur Teilnahme an diesem Symposium bedanken. Mein Dank gilt hier auch allen Teilnehmern, deren vielseitige Beiträge, kritische Fragen und Kommentare ungemein inspirierend waren. Mein Dank gilt hier insbesondere Prof. Dr. Walther Sallaberger und PD Dr. Peter-Arnold Mumm. Ohne deren intensive und konstruktive Kritik wäre dieser Beitrag in dieser Form nicht möglich gewesen. Weiter geht besonderer Dank an Frau Nora Kuch (M.A.) für ihre Hilfe bei der Erstellung und Bearbeitung des Bildmaterials und ihrer ausführlichen Korrekturen des Textes in allen Entwicklungsstadien.
}

Ә Open Access. () 2018 Fabian Heil, publiziert von De Gruyter. (cc) BY-NC-ND Dieses Werk ist lizenziert unter der Creative Commons Attribution-NonCommercial-NoDerivatives 4.0 Lizenz.

https://doi.org/10.1515/9783110601268-005 
wird in dieser prähistorischen Archäologie nicht länger ernsthaft verwendet, zumindest falls keine entsprechende Selbstbezeichnung aus Schriftquellen vorliegt. Stattdessen hat sich der neutrale Begriff der "archäologischen Kultur“ in der Forschung etabliert. Wie rechtfertigt sich nun ein Beitrag zum Thema von „Volk und Sprache“ aus Sicht der prähistorischen Archäologie? Die Antwort darauf ist eine weitere Frage und betrifft den Umgang der prähistorischen Forschung mit dem Kulturbegriff selbst: ist er tatsächlich grundlegend anders konzipiert als die alte Idee eines „Volkes“ oder ist er nur der Versuch einer Neuauflage, um sich von einem negativ konnotierten Begriff zu befreien? In Peter-Arnold Mumms Einführung zu diesem Buch wurde bereits dargelegt, dass und wie ein pauschalisierter Kulturbegriff die Beschreibung einer Gesellschaft einschränkt. Wenn aber zwischen Kultur und Gemeinschaft unterschieden wird, sind sowohl die interagierenden Individuen als auch die sie umgebenden „Dinge“ in ihrem reziproken Abhängigkeitsverhältnis relevant und verweisen auf verschiedene Aspekte zwischenmenschlicher Interaktion und gemeinschaftlicher Selbstdarstellung. Auch wenn sich bereits einige entsprechende Konzepte in den Kulturwissenschaften erfolgreich etabliert haben, hat eine solche differenzierende Sichtweise in der prähistorischen Archäologie leider noch nicht überall Fuß gefasst.

Dieser Beitrag soll anhand des vorzustellenden Beispiels der zyprischen Bronzezeit aufzeigen, dass innerhalb der Zypernforschung nicht nur die Vorstellung eines „Volkes“ erhalten geblieben ist, sondern auch Erklärungsmuster von diesem Prinzip unbewusst abgeleitet werden. Der Beitrag stellt ausgewählte Funde und Befunde vor, die eng mit dem Prinzip der Hybridisierung in Verbindung stehen - ein besonders prominenter Ansatz für die Bewertung einer „zyprischen Kultur“. Durch eine Schwerpunktverlagerung in der Betrachtungsweise werden schließlich alternative Deutungsmöglichkeiten angeboten.

In der prähistorischen Archäologie ist die Hauptquelle jeder Argumentation über Kultur das Artefakt und dessen Kontext. Um die Fülle aller auffindbaren Artefakte zu überblicken, ist eine quantitative Beschränkung für einen Vergleich notwendig. Diese wird üblicherweise in dem Versuch einer räumlichen und zeitlichen Eingrenzung eines Arbeitsgebietes anhand einer Kultur vorgenommen, wobei Kultur als „die Gesamtheit der überwiegend sozial, d. h. nicht genetisch tradierten menschlichen Lebensäußerungen “1 verstanden werden sollte. Mit einer Zusammenstellung der daraus überlieferten Artefakte, also durch menschliches Wirken erschaffenen Dinge, können so zumindest einige der durch soziale Interaktion tradierten Lebensäußerungen von Menschen-

1 Wotzka (2000) 55. 
gruppen erfasst und darauf aufbauend das Leben prähistorischer Menschengruppen und deren Interaktion untereinander beschrieben werden. ${ }^{2}$ Betrachtet man nun bereits durch Prähistoriker definierte ,archäologische Kulturen“, fällt sehr schnell auf, dass deren Benennung, beispielsweise die der neolithischen Trichterbecherkultur, der mittelbronzezeitlichen Hügelgräberkultur oder der mykenischen Kultur dabei auf unterschiedlichen Hauptkriterien beruhen. Entweder wurden herausragende Keramiktypen als Namensgeber ausgewählt, eine bestimmte (Bestattungs-)Sitte oder auch der erste (oder prominenteste) Fundort. Diese Benennungen basieren also auf einer retrospektiv durchgeführten Auswahl und sind nicht zwingend auch als hochrangiges, identitätsstiftendes Element der jeweiligen Kulturträger $\mathrm{zu}$ verstehen. Neben diesen Hauptmerkmalen werden auch weitere Charakteristika für die Unterscheidung dieser archäologischen Kulturen herangezogen, die aber nicht zwingend deckungsgleich mit allen materiellen Äußerungen im Arbeitsgebiet auftreten. Daher ist eine „,archäologische Kultur“, vereinfacht ausgedrückt, ein unspezifisches „Merkmalbündel“ verschiedener zusammen auftretender, materieller Hinterlassenschaften (Gefäßformen, Bestattungsweise, Architektur, usw.) und bildet somit einen Hilfsbegriff, aber zugleich auch den Grundbaustein für die Erstellung regionaler archäologischer Sequenzen, ohne dabei aber eine soziale oder politische Einheit der zeitgenössischen Kulturträger zu postulieren. ${ }^{3}$

Ein einheitliches Kulturkonzept hat die prähistorische Archäologie dabei aber noch nicht gefunden. Dies zeigt beispielsweise Manfred Eggert anhand der Arbeiten von Alfred Louis Kroeber und Clyde Kluckhohn (1963), die mit ihrer eindrucksvollen Zusammenstellung von über 300 Definitionen belegen, wie unterschiedlich dieser Begriff verstanden werden kann. ${ }^{4}$ Die Entwicklungen, Probleme und Stärken der verschiedenen Definitionsansätze können hier nicht erschöpfend diskutiert werden. ${ }^{5}$ Es seien hier nur einige Hauptstationen der Kontroverse herausgegriffen. Den vermutlich am häufigsten zitierten Ansatz stellt der „konventionelle Kulturbegriff“ dar, der von Gordon Childe zuerst sehr klar formuliert wurde und „Kultur“ zu einem etablierten terminus technicus in der Prähistorie machte:

„We find certain types of remains - pots, implements, ornaments, burial rites, house forms - constantly recurring together. Such a complex of associated traits we shall term a 'cultural group' or just a 'culture'. We assume that such a complex is the material expression of what would to-day be called a 'people'. 6

2 Wotzka (2000) 58.

3 Wotzka (1993) 33-34; Eggert (2013) 14.

4 Eggert (2013) 15.

5 Siehe dazu Eggert (2013).

6 Childe (1929) V-VI; Wotzka (1993) 30. 
Nach dieser frühen Definition Childes war also anhand regelmäßig zusammen auftretender materieller Hinterlassenschaften nicht nur eine lose Gemeinschaft definierbar, sondern es war auch die Möglichkeit gegeben, durch große Ähnlichkeiten im Material eine politisch und sozial (aber, explizit, nicht-rassisch) zusammengehörige Kulturgruppe erkennen zu können. Aber „Realien“, also Funde und Befunde, sind nach diesem Verständnis in hohem Maße als allzu selbstverständliche Indizien eines dahinterstehenden „Volks“ zu verstehen und bewirken bei einem undifferenzierten Kultverständnis einen argumentativen Zirkelschluss. Denn Objekte reichen für sich alleine stehend nicht aus, um Menschengruppen als Kultur archäologisch fassen zu können. Erst die damit in Verbindung stehenden Handlungen sind es, die überhaupt einen Einblick auf Kulturen erlauben. Eggert versteht dieses Problem einer aus Realien abgeleiteten Selbstevidenz daher als die positivistische Konstruktion archäologischer Fakten. ${ }^{7}$ Zwar korrigierte sogar Childe selbst seinen soziologisch gemeinten Ersatz von „races“ durch „peoples“ mehrfach, um sich weiter von essentialistischen Kulturvorstellungen zu entfernen, und stellte sich dabei zeitlebens bewusst gegen rassische Projektionen, ${ }^{8}$ doch die einprägsame Formulierung früherer Entwürfe führt bis heute zu unreflektierten Rückgriffen.

Eine andere Herangehensweise ist der „historisch-diffusionistische Kulturbegriff“. Dieser erweitert zwar die frühesten Ansätze Childes, versteht die kulturellen Aneignungsprozesse aber nur begrenzt als komplexe Entwicklungsprozesse sozialer Interaktion. Stattdessen werden sie als die Folge „zivilisatorischen Drängens" 9 verstanden, das dem menschlichen Verlangen nach Fortschritt und den wirtschaftlichen Auswirkungen technologisch/kultureller Überlegenheit entspringt; ein Ansatz, der aber immer weniger überzeugend wirkt, ,je weiter sich Natur- und Geisteswissenschaft ausdifferenzieren“ ${ }^{10}$

Der „funktionalistische Kulturbegriff“ hingegen lehnt es nach Eggert ab, Ähnlichkeiten innerhalb materieller Hinterlassenschaften bereits als ausreichenden Beleg für eine darin abgebildete gesellschaftliche Gruppierung zu werten. Er begreift die Kultur nicht nur als materiell fassbar, sondern als ein dem Menschen eigenes „Artmerkmal“ mit dem Potential, unterschiedlichste Gesellschaftsstrukturen zu entwickeln. Es reicht in der Folge nicht aus, bestimmte Äußerlichkeiten von Objekten einem bestimmten Typus oder auch definierten Gruppen von Menschen zuzuweisen. Dabei wird die Idee vertreten, dass auch das in der Archäologie übliche Kartieren und Addieren von Kultur-

7 Eggert (2013) 31.

8 Childe (1933); (1956) 112; (1963) 40-60; Tringham (1983).

9 Eggert (2013) 33. Siehe besonders Fußnote 43.

10 Bräunlein (2012) 17. 
elementen nicht objektiv geschehen kann. Denn bereits die Auswahl des dargestellten Materials leidet oft an einer eher rudimentären Dokumentation und einem beschränkten Informationsgehalt, überwiegend in Bezug auf deren Erscheinung und Äußerlichkeiten. Entsprechen sind Merkmalkataloge oder Kartierungen stets aufgrund methodischer Filter vorsortiert. Kartierte Objektgattungen stellen daher nur ein relativ grobes Werkzeug der Archäologie dar, um den Zugriff auf ausgewählte Charakteristiken und Objektgruppen in einem bestimmten Raum zu einer bestimmten Zeit abzubilden und diese $\mathrm{zu}$ kommunizieren. Dieser Darstellungsweise ist es auch geschuldet, dass die Bedeutung oder Behandlung dieser innerhalb des jeweiligen Kontextes kaum eine große Rolle spielen kann. Dieses Vorgehen reicht entsprechend nicht aus, um daraus eine selbstständige Kulturgruppe ableiten zu können. Stattdessen müssen die unzähligen Realisierungen der Kultur im Material als Teile übergeordneter Wirkungszusammenhänge verstanden werden. Dabei wird die enge Verbindung der Dinge mit der soziokulturellen Identität des Menschen betont, so dass eine („materielle“) Kultur (s. u. Zitat Hahn) keine eigene Größe darstellt, die es von anderen Kulturen zu unterscheiden gilt, sondern sie ist Ausdruck soziokultureller Beziehungen. ${ }^{11}$ So wird die Notwendigkeit betont, nicht nur eine „beschreibende Bestandsaufnahme“ des archäologischen Materials zu liefern, sondern auch einen Einblick in die soziokulturelle Entfaltung der Menschheit zu gewinnen und deren mittel- und langfristige Abläufe innerhalb von Gesellschaften $\mathrm{zu}$ überblicken, egal welcher Fokus im konkreten Fall Gegenstand einer Studie sein mag. ${ }^{12}$ Um dies zu erreichen, bedarf es eines Kulturverständnisses jenseits des „traditionellen“, ethnisch orientierten Ansatzes, das eine Zusammenarbeit mit anderen Kultur- und Geisteswissenschaften voraussetzt. Um die Archäologie nicht auf die wissenschaftliche Praxis des Sammelns zu begrenzen, sind Disziplinen in den archäologischen Diskurs einzubinden, die menschliche Interaktion zu ihrem Schwerpunkt gemacht haben. Dies wird beispielsweise innerhalb des ,semiotisch-kommunikationstheoretischen Kulturbegriffes“ vertreten. Dieser Ansatz geht von einer „materiellen Kultur“ aus, der „Summe aller Einzelgegenstände einer Gesellschaft [...], die benutzt oder bedeutungsvoll sind“, ${ }^{13}$ wobei nicht der Grad ihrer Bedeutung von Belang ist, sondern die Tatsache, dass sie überhaupt verwendet wurden. ${ }^{14}$ Nach Hahn resultiert die polyvalente Bedeutung dieser Dinge nicht aus ihrer Materialität, sondern dem Kontext ihrer Verwendung und der damit zu verknüpfenden Kon-

11 Bräunlein (2012) 21.

12 Eggert (2013) 35.

13 Hahn (2005) 18.

14 Eggert (2013) 38. 
zeptualisierung der Anwender. ${ }^{15}$ Dies geschieht unter Berücksichtigung aller sozialen Interaktion, so dass „Kultur“ von Andreas Wimmer als offener und instabiler Vorgang des Aushandelns verstanden wird, als „kultureller Kompromiss“. ${ }^{16}$ Im Kontext der archäologischen Fragestellung sind daher nach Wimmer (1996) verschiedene Ebenen des Kulturkonzeptes zu berücksichtigen.

1. Erlaubt das Kulturkonzept einen Einblick in die verinnerlichte Kultur eines Individuums?

2. Gibt das Kulturkonzept Hinweise auf eine allgemeine, verbindliche Vorstellung über die Beschaffenheit der Welt?

3. Schließt das Kulturkonzept kulturelle Praktiken ein, die Grenzen sozialer Gruppen markieren, innerhalb derer ein Aushandlungsprozess stattfindet?

Auf die Fragen zwei und drei kann anhand von Beispielen der zyprischen Spätbronzezeit durchaus ausführlich Bezug genommen werden (s. u. § 4). Dabei gilt es zu diskutieren, ob beispielweise ein Keramikstil, Bestattungssitten oder Ähnlichkeiten in der Architektur auf ein indigenes Gemeinschaftsempfinden hinweisen und damit Rückschlüsse auf ähnliche soziale Strukturen erlauben. Die erste Frage hingegen entspricht zwar den Prinzipien des Habitus und dem Antrieb des menschlichen Handelns, aber ohne ausführliche Schriftquellen ist diese im archäologischen Befund kaum bis nicht fassbar und kann nur übergeordnete, soziologische Fragestellung bedienen. ${ }^{17}$ Ob dabei im Einzelfall von einem inneren Gemeinschaftsgefühl gesprochen werden kann, unterliegt in der archäologischen Forschung dabei extremer Uneinigkeit. Da viele der unzähligen zwischenmenschlichen Faktoren in emischer Perspektive leider nicht mehr direkt nachvollziehbar sind, muss auf theoretische Konzepte aus sozialanthropologischen Disziplinen, der Kognitionswissenschaft oder auch der Semiotik zurückgegriffen werden. Nur so können archäologisch erfasste Daten nicht nur als typologische Sequenzen verarbeitet und auf Verbreitungskarten dargestellt werden, sondern man kann auch den Transport kultureller Inhalte analysieren. Denn dieser findet nicht nur verbal, bzw. durch Beobachtung und Nachahmung während sozialer Handlungen statt, sondern steht in direkter Relation zur Handhabung, Herstellung und Interaktion mit und von Objekten. Durch diese Rück- und Wechselwirkung steht die „Kultur“ also im Sinne dialektischer Beziehungen zwischen Kognition, sozialer Praxis und den von Menschen geschaffenen materiellen Dingen. ${ }^{18}$ Durch diese Abstraktion des Kulturbegriffes

15 Eggert (2013) 38 Fußnote 56.

16 Wimmer (2011) 415.

17 Wimmer (1996) 407-408.

18 Wotzka (2000) 58. 
können somit zahlreiche komplexe und wechselseitige Charakteristiken in unterschiedlicher Kombination berücksichtigt werden, wie beispielsweise Kunst, Ernährung, Wirtschaft, Kleidung, soziopolitische Ordnung, Religion/Kult/Symbolik, Außenbeziehungen, usw.; man muss dafür nicht zwingend einen „Stamm“ oder ein „Volk“ postulieren. ${ }^{19}$

Unter Berücksichtigung des ethnologischen Kulturverständnisses, das eine traditionelle Definition des Begriffs „Kultur“ als unverwechselbare und historisch dauerhafte Einheit ablehnt, muss von einer Homogenitätsvorstellung auch innerhalb einer archäologischen Kultur gänzlich Abstand genommen werden und intrakulturelle Variation als grundlegend für selbst einfachste Gemeinschaften betont werden. ${ }^{20}$ Damit wäre die „archäologische Kultur“ also einzig als statistisches Konstrukt zu verstehen. ${ }^{21}$ Diese Schlussfolgerung erklärt, dass es heute ein Bestandteil fachinterner Debatten ist, ob der Begriff der „Kultur“ und alle damit im Zusammenhang stehenden Synonyme und Ausweichbegriffe (Stamm, Volk, Ethnizität, etc.) vollständig zu vermeiden sind oder ob man diesen ethnologischen Ansatz meiden soll und weiterhin die „Kultur“ als reale, im Material erkennbare historische Gemeinschaft suchen kann. ${ }^{22}$ Welcher Ansatz nun gewählt wird, liegt schlussendlich im Ermessen des Wissenschaftlers. Doch im weiteren Verlauf dieses Beitrages wird anhand des Beispiels der zyprischen Archäologie auf Probleme eingegangen werden, die durch die Annahme erwachsen, eine ,reale, historische Gemeinschaft“ auf Zypern erkennen zu können.

\section{Zypern als „hybride“ Gesellschaft?}

Die vorgestellte Problematik, die mit der Verwendung des Kulturbegriffes in der Archäologie einhergeht, wird in der zyprischen Archäologie nur selten diskutiert. Im Gegenteil, mit der Wahl des Titels „Cyprus. Crossroads of Civilizations“ machte eine im Smithsonian Museum of Natural History ausgerichtete Ausstellung aus dem Jahre 2010 besonders auf eine diskussionswürdige Facette der zyprischen Archäologie aufmerksam: die Nachweisbarkeit einer Akkumulation kultureller Einflüsse auf Zypern, sowohl aus dem östlichen als auch dem zentralen mediterranen Raum. ${ }^{23}$ Der Höhepunkt dieser Einflussnahme

19 Wotzka (2000) 56 Abb. 1.

20 Bricker Reifler (1975).

21 Wimmer (1996) 403-404.

22 Lüning (1972) 169; Lichardus (1991a) 13.

23 Hadjisavvas (2010). 
liegt vermutlich im 11. Jh. v. Chr. und ist durch intensive Austauschbeziehungen mit den etablierten Großreichen Ägypten, des mykenischen Griechenlands und den levantinischen Stadtstaaten zu erklären. Für diese Zeit steht die steigende Präsenz von Objekten, Formen und Ideen, die offenbar mit dem griechischen Festland in Verbindung stehen, im Vordergrund der Forschungsdiskussion. Eine herausragende Position nimmt diesbezüglich die griechische Sprache ein. Der Fund eines beschrifteten Obelos (no. 16), einer Art Bratspieß, aus Grab 49 bei Palaipaphos-Skales ${ }^{24}$ zeigt scheinbar anhand des Namens o-pe-le-

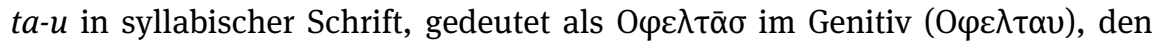
Wandel von - $o$ zu $-u$ an der Wortendung, einem Charakteristikum des klassischen, zyprischen Griechisch, das damit die Nähe zum festlandgriechischen Arkadischen und dem mykenischen Griechisch der Linear-B Schrift belegen soll. ${ }^{25}$ Besonders interessant ist aber, dass das arkadische Griechisch hier nicht in einem Kontext einer mykenisch oder griechisch anmutenden Grabanlage nachgewiesen ist, sondern die Niederlegung streng nach lokalen Sitten gestaltet wurde. Es ist also nicht erkennbar, ob sich der Name auf dem Spieß auf eine Person griechischer Herkunft bezieht, aber die Präsenz der Sprache und deren Eigenheiten ist mit Sicherheit bereits zu dieser Zeit auf Zypern verbreitet und vermutlich auch bereits vorher anzunehmen (Abb. 1). ${ }^{26}$ Ergänzend wird anhand ausgewählter Objekte im Folgenden eine ähnliche Verbindung zu Regionen außerhalb Zyperns aufgezeigt.

Interaktion lässt sich archäologisch einerseits aufgrund des Auftretens von Importen und Exporten nachweisen, andererseits aber auch anhand typologischer Charakteristika. Ein Import zeigt aber nur an, dass ein Objekt über große Entfernungen bewegt wurde. Die Dauer, diese zu überbrücken, bleibt dabei jedoch genauso unklar, wie die verschiedenen Stationen des Transports, die Intention und Art des Austausches und auch die Anzahl an Individuen, die mit dem Objekt tatsächlich Kontakt hatten. Ein ägyptisches Gefäß der Zweiten Zwischenzeit innerhalb eines spätbronzezeitlichen Grabes auf Zypern bezeugt also nicht zwingend die Interaktion zwischen Ägypten und Zypern, sondern könnte auch über die Levante, Anatolien oder sogar Kreta abgelaufen sein. So bleibt unklar, welche Assoziationen damit übertragen wurden und ob mehr als die bloße Andersartigkeit des Äußeren als relevant wahrgenommen wurde.

Typologische Gemeinsamkeiten hingegen bezeugen tiefergehende Interaktion. Ähnlichkeiten in der Formgebung und Handhabe eines Gegenstandes

24 Karageorghis (1983) 61.

25 Ausführlich dazu Deger-Jalkotzy (1994) 11; Kritik an dieser Deutung zudem in Olivier (2013); Egetmeyer (2013); Steele (2016).

26 Steele (2016) 13-15. 


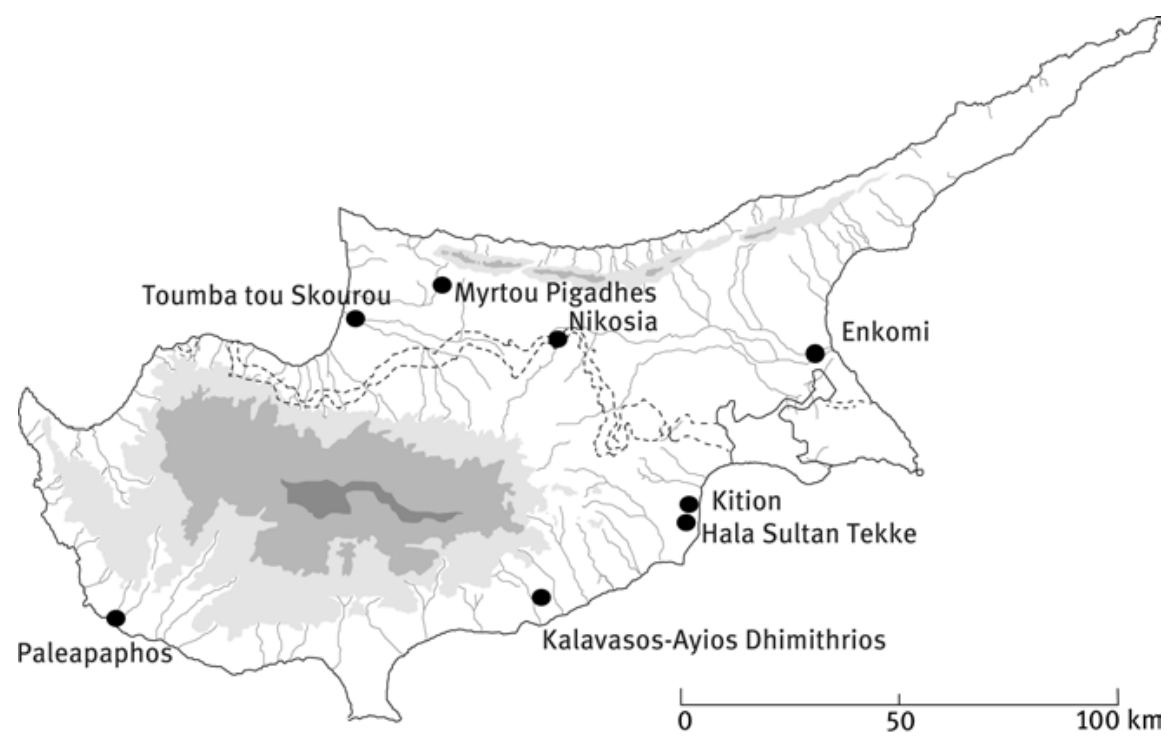

Abb. 1: Übersichtskarte aller im Text erwähnten Fundorte auf Zypern. Gestrichelt markiert ist die moderne UN-Pufferzone zwischen der Republik Zypern und der Türkischen Republik Nordzypern.

deuten auf einen kontextuell vorbestimmten Inspirations- bzw. Interaktionsraum hin - beispielsweise eine Werkstatt -, innerhalb dessen Individuen mit unterschiedlichen Erfahrungsschätzen - beispielsweise aufgrund von Herkunft - kommunizierten. So wird nicht nur ein Objekt ausgetauscht, sondern auch Ideen, Vorstellungen und Meinungen. Nur so wird die Interaktion mit umgebenden Kulturen offensichtlich. Das Fehlen solcher Prozesse belegt aber nicht im Umkehrschluss das Fehlen von Interaktion.

Besonderheiten dieser Art führen jedoch auch zu einer überzogenen Erwartungshaltung in der Forschung: Man erwartet von vornherein, dass Objekte innerhalb der zyprischen Archäologie bereits als kulturimmanentes Charakteristikum einen „Mischcharakter“ in sich tragen. Geringe Beachtung erfährt dabei das Konzept der kulturellen Aneignung, das kulturspezifische Formen des Erwerbs, „Einbrauchens“, Umarbeitens, Konsumierens und damit einhergehender Veränderungen der Wahrnehmung näher beschreibt. ${ }^{27}$ Dabei liegt das Problem im methodischen Vorgang der archäologischen Kategorisierung,

27 Schreiber (2013) 13. 
durch die eine Projektion eigener Erwartungen und (wissenschaftlicher) Kategorien auf antike Kulturen besteht. ${ }^{28}$ Es entspricht den etablierten Methoden in der Archäologie, lokale Strukturen, Materialien und auch Verhaltensmuster mit umgebenden Kulturen zu vergleichen, wie es auch in der zyprischen Archäologie geschehen ist und in Anbetracht der geographischen Trennung vom Festland auch sinnvoll ist. Die dabei identifizierten Ähnlichkeiten und unterschiedlich gearteten Verbindungen wechselnder Einflüsse sind unbestreitbar. Diese werden in der Forschung dabei aber als „Hybride“ bezeichnet, ein Begriff, der als methodische Notwendigkeit auf Objekte projiziert wird, die offensichtlich stilistische Merkmale anderer Kulturen aufweisen und so nicht einer einzigen Kategorie zuzuweisen sind. Dabei unterstellt dieser Begriff, dass es eine Form von „reinen“ Archetypen und im Umkehrschluss von degenerierten und unterlegenen Elementen gäbe. ${ }^{29}$ Doch während die Verwendung des Begriffes der Hybridität eigentlich vor allem im Diskurs der post-kolonialen Zeit ein politisches Ziel hatte - damit wurde bewusst versucht, verschiedene Identitäten und widersprüchliche Ideen zusammenzuführen - können derartige Rahmenbedingungen nicht für das prähistorische Zypern angenommen werden. ${ }^{30}$ Durch die scheinbar extraordinäre Quantität und Qualität des kulturellen Einflusses wird die zyprische Kultur aber selbst als eine Art Hybrid verstanden. ${ }^{31}$ Dabei erwächst der Eindruck, als habe Zypern in der Spätbronzezeit keine eigenständige Kultur, sondern stelle eine Art kulturelles Konglomerat dar, also eine verbackene Mischform verschiedener kultureller Einflüsse aus Griechenland, Anatolien, Ägypten und der Levante, die sich aus dem archäologischen Material ablesen lasse und die sich auf der Mittelmeerinsel akkumuliert habe:

In contrast to the apparent isolation of earlier periods, the L[ate] C[ypriot] period is characterized by the assimilation and adaption of foreign iconography and technologies. Hybridisation is apparent in many aspects of the LC cultural repertoire $[. . .]^{32}$

Offenbar werden insbesondere durch die Annahme, Phasen der Isolation erkennen zu können, so genannte Hybridisierungsvorgänge innerhalb von Technologie und Ikonographie zur wichtigsten Referenz der Forschung. Durch neue Diskussionen zur Hybridität (Stockhammer 2013) wird zudem die Möglichkeit wieder in Betracht gezogen, anhand typologischer Charakteristiken differen-

28 Sherratt (2015) 75.

29 Ackermann (2012) 6.

30 van Dommelen \& Rowlands (2012); Hodos (2006) 16-17.

31 Matthäus (2000) 91.

32 Steel (2004a) 169. 
zierbare Gemeinschaften postulieren zu können, wodurch das Kulturproblem „[...] durch die Hintertür“ erneut auftritt. ${ }^{33}$

Für die Interpretation sogenannter hybrider Objekte bleibt aber die Betrachtungsweise des Materials entsprechend dem Konzept der materiellen Kultur nach Hahn notwendig (s. o.). Nutzen oder Bedeutung eines Gegenstandes sind ständiger Veränderung unterworfen und von einer bestimmten Zeit, kontextuellen Nutzungsphasen und von der jeweiligen individuellen Rezeption abhängig. Die Bedeutungszuschreibungen werden dabei mit Bekanntem verglichen oder gänzlich neuen Kategorien zugeordnet, wenn sich keine entsprechende Korrespondenz finden lässt. Demzufolge „denken Menschen durch materielle Kultur“. ${ }^{34}$ Es ist also nicht nur das archäologische Material selbst von Interesse, sondern auch wie es Verwendung fand, um $\mathrm{zu}$ ergründen welche spezifischen Bedeutungen kontextbedingt zu berücksichtigen sind. ${ }^{35}$ Das Kombinieren von Objekten und Ideen ist etwas Alltägliches und wird durch eine Vielzahl verschiedener Quellen beeinflusst, durch Werkzeug, Rohstoff, sozialen Habitus und der Persönlichkeit des Akteurs selbst. ${ }^{36}$ Diese Erkenntnis ist deshalb wichtig, da sich bereits (berechtigter) Widerstand in der Zypernforschung bezüglich der verwendeten Terminologien regt. ${ }^{37}$ Tatsächlich stehen sich verschiedene Ansätze gegenüber, wie mit der Frage einer Herausbildung einer einheitlichen Kultur am Ende der Spätbronzezeit auf Zypern umzugehen ist. Es stellt sich die Frage, wie von dieser einheitlichen Kultur am Ende der Spätbronzezeit gesprochen werden kann, wenn zugleich die Entwicklung dieser Kultur aus einer Vielzahl externer Einflüsse heraus geschehen sein soll. Dieser Ansatz suggeriert nicht nur eine gemeinsame kulturelle Identität als Ergebnis von Handel und Austausch, sondern auch eine gleichgeschaltete Adaption „fremder“ Sitten durch die Bewohner der Insel. Auch Bernhard Knapp möchte in diesem Sinne von einer Hybridisierung anstelle einer Akkulturation sprechen, Crewe hingegen hält eine derartige nuancierte Differenzierung zumindest in Hinblick auf die Formierungsprozesse (zumindest während der frühbronzezeitlichen Philia Phase, siehe Tab. 1) für überflüssig. ${ }^{38}$ Sie bringt dabei jedoch einen elementaren Ansatz nicht zur Sprache: die Annahme einer hybriden Kultur führt in ihrer Folge zur Definition einer Art von reinem Archetypen, der kritisch zu sehen ist:

33 Maran (2012).

34 Hodder (2012) 35.

35 Wulf (2011) 83.

36 Bourdieu (1990) 54-55.

37 Crewe (2014) 137.

38 Knapp (2013a) 266-268; 454-455; Crewe (2014) 137. 
[...] the identification of »hybridity« itself [is] dependent on the same essentialist framework that it seeks to challenge. In order for us to identify something as »hybrid«, we need to place it within a framework of what, at best, are our own artificial and agenda-laden archaeological categories and, at worst, may be effectively essentialist classifications and definitions. ${ }^{39}$

Der Begriff „Hybridität“ ist also nur mit Vorsicht zu gebrauchen. Er suggeriert eine Abkehr vom „Volks“-Gedanken, lässt aber die Frage offen, was sich kreuzt. Relevant ist diese Frage, wenn ein bestimmtes Objekt analysiert wird und zu entscheiden ist, ob ein ikonographisches Element übernommen wurde oder eine Bedeutung, die mit einem Attribut zusammenhängt, oder ob das Element womöglich nur mit Hinblick auf einen bestimmten Verwendungszweck zu deuten ist. Sofern aber an essentialistische Kulturen gedacht wird, die hier in einen „Schmelztiegel“ geraten wären, bleibt der Hybriditätsbegriff ganz im alten Paradigma.

Das Problem macht damit nicht nur eine saubere Kategorisierung der archäologischen Daten schwierig, sondern wirkt sich direkt auf die Aussagekraft jedweden archäologischen Materials aufgrund der stets durch Empirie erfassten Daten aus. Denn auch wenn eine Objektgruppe einer bestimmten, klar umrissenen Kultur als zugehörig verstanden werden kann, verklärt die offenbare Verschmelzung bestimmter Elemente, die eigentlich räumlich voneinander getrennt erfasst werden können, diesen Umstand als das Ergebnis einer scheinbar umfassenden Interaktion. Dabei ist aber klar, dass auch dabei immer eine Selektion stattgefunden hat. Denn immerhin werden stets nur ganz bestimmte Elemente miteinander verbunden.

\section{Eine kulturhistorische Einführung}

Die Forschung über die Bronzezeit Zyperns ist durch dieses Problem der kulturellen Differenzierung in einer Vielzahl von Widersprüchen verfangen. Der Beginn der Bronzezeit wird durch einen inselweit fassbaren Wandel in der materiellen Kultur definiert, der nur durch Kontakte an das umgebende Festland möglich war. Zugleich sind daraufhin klar erkennbare Importe - eine der archäologischen Hauptquellen für Fernkontakte - extrem selten und erst mit der Spätbronzezeit zahlreich genug, um von einer frequentierten Austauschbeziehung ausgehen $\mathrm{zu}^{40}$ So wird weiterhin postuliert, dass es lange Phasen der

39 Sherratt (2015) 75.

40 Mina (2013); Antoniadou (2003). 
Isolation gegeben habe, die erst mit der auslaufenden mittleren Bronzezeit gegen 1700 v. Chr. dauerhaft geendet hätten (s. u. Tab. 1). ${ }^{41}$

Diese Verschiebungen und das Fehlen von Importen führten also zur Gegenüberstellung alternierender Phasen der Interaktion und Isolation, dem scheinbar unentwegten Aufflammen und Verebben der Interaktionsbestrebung.

Doch es gibt auch eine andere Möglichkeit, dieses Phänomen zu erklären. Man muss nämlich in Betracht ziehen, dass die Objekte das Resultat von Austauschmechanismen sein können, die kulturspezifischen Regeln und Einschränkungen unterworfen sind, die eine Limitierung von Interaktionsräumen, Anwendungen und Austausch verursachen. Anstelle scheinbar liberal gestalteten Austauschs und Handels ist dann von strengen Regulierungen und verbindlichen Rahmenbedingungen auszugehen. Der auch schriftlich belegte Austausch von politischen Tributen oder Geschenken liefert entsprechende Rahmenbedingungen, zumindest auf einem sehr elaborierten Level. ${ }^{42}$ Diese Erklärung geht von der Prämisse aus, Importe nicht einzig als eine Art von Symbol für eine entfernte Kulturerscheinung und in der Folge als Beleg für materiellen Austausch/Handel zu begreifen. Importe sind stattdessen auch unabhängig von ihrer Quantität zu bewerten. Die Möglichkeit, dass die Bewohner der Insel stets im Kontakt zu anderen Kulturen des Mittelmeerraums standen, muss eine valide Option darstellen, sofern die technologischen Grundvoraussetzungen dafür gegeben waren. Wie sich diese Interaktion gestaltete, war aber auch stetem Wandel unterworfen; ein stetes Verschieben einer kulturell geprägten Rahmung ist als Teil des kulturellen Kompromisses anzunehmen. Denn auch im weiteren Verlauf der Geschichte sind die Bewohner der Insel stets in kulturellem Austausch integriert, der sich auch aktuell in gesellschaftlichen Zerwürfnissen auf der seit 1974 zweigeteilten Insel widerspiegelt. ${ }^{43}$

Zentral für die mögliche Einflussnahme ist also, wie die Qualität des Austausches $\mathrm{zu}$ bewerten ist. Der bisherige Konsens ist, das Ansteigen der spätbronzezeitlichen Importe stets als Ergebnis eines umfassenden und zunehmend intensivierten kulturellen Austausches mit dem minoischen Kreta, dem mykenischen Griechenland, dem hethitischen Großreich in Anatolien, Ägypten und den levantinischen Stadtstaaten zu begründen. Dieser gehe mit einer „dramatischen Transformation durch Kontakte mit und die Assimilation von externen kulturellen Einflüssen“ einher und habe die Gesellschaft auf der Insel ver-

41 Steel (2004a) 143; Peltenburg (1996).

42 Panagiotopoulos (2012) 53-55.

43 Matthäus (2000) 91; Stevens (2016) 21-25. 
Tab. 1: Chronologietabelle der zyprischen Bronzezeit. $F Z=$ Frühzyprisch, $M Z=$ Mittelzyprisch, $\mathrm{SZ}=$ Spätzyprisch, ZG = Zypro-Geometrisch, PreBA = Prehistoric Bronze Age, ProBA = Protohistoric Bronze Age.

\begin{tabular}{|c|c|c|c|}
\hline \multirow{2}{*}{$\begin{array}{l}\text { Absolute Chronologie } \\
\text { v. Chr. (nach Knapp 2013a } \\
\text { and Steel 2010) }\end{array}$} & \multicolumn{2}{|c|}{ Relative Chronologie } & \multirow{2}{*}{$\begin{array}{l}\text { Absolute Chronologie } \\
\text { v. Chr. (nach Merrillees } \\
1992 \text { und Manning et al. } \\
\text { 2001) }\end{array}$} \\
\hline & $\begin{array}{l}\text { Alternative } \\
\text { (Knapp 1994) }\end{array}$ & Traditionell & \\
\hline $2500 / 2400-2350-2250$ & \multirow[t]{4}{*}{ PreBA I } & Phila & $2500-2300$ \\
\hline \multirow[t]{4}{*}{$2350 / 2250-2000$} & & FZ I & $2300-2150$ \\
\hline & & FZ II & $2150-2100$ \\
\hline & & FZ IIIA & $2100-1950$ \\
\hline & \multirow[t]{3}{*}{ PreBA II } & FZ IIIB & $2025-1950$ \\
\hline \multirow[t]{2}{*}{$2000-1750 / 1700$} & & MZ I & $1950-1850$ \\
\hline & & MZ II & $1850-1750$ \\
\hline \multirow{3}{*}{$\begin{array}{l}\text { 1750/1700-1450 } \\
\text { (Übergang) }\end{array}$} & \multirow[t]{3}{*}{ ProBA I } & MZ III & $1750-1650$ \\
\hline & & SZ IA & $1650-1550$ \\
\hline & & SZ IB & $1550-1425$ \\
\hline \multirow[t]{3}{*}{$1450-1300 / 1200$} & \multirow[t]{3}{*}{ ProBA II } & SZ IIA & $1425-1375$ \\
\hline & & SZ IIB & $1375-1330$ \\
\hline & & SZ IIC & $1330-1200$ \\
\hline \multirow[t]{3}{*}{$1300 / 1200-1050$} & \multirow[t]{3}{*}{ ProBA III } & SZ IIIA & $1200-1100$ \\
\hline & & SZ IIIB & $1100-1050$ \\
\hline & & SZ IIIC/ZG I & $1050-1000$ \\
\hline
\end{tabular}

ändert. ${ }^{44}$ Denn während dieses Abschnittes der mittleren und beginnenden späten Bronzezeit (Mittelzyprisch III - Spätzyprisch I, ca. 1700-1600 v. Chr.) steigt die Präsenz zyprischen Materials im Austauschsystem des mediterranen Raumes deutlich an. So sei eine Integration Zyperns in den weitreichenden mediterranen Handel durch den Austausch von Ressourcen, Waren und Ideen im archäologischen Material auf vielfältige Weise fassbar. ${ }^{45}$ Da Zypern zu dieser Zeit (noch) als wichtigster Bezugsort für Kupfer im mediterranen Raum gilt,

44 Steel (2004a) 142.

45 Sherratt (2015) 76. 
werden wirtschaftliche Interessen als einer der Hauptgründe für die Intensivierung des Austausches genannt. ${ }^{46}$ Durch die handelsbasierte Integration der Insel in das umfassende Austauschsystem der Großmächte des östlichen mediterranen Raumes sei demnach eine weitreichende Neustrukturierung der Gesellschaft auf Zypern erkennbar, die von einer zuvor „egalitär-agrarwirtschaftlichen “ Gesellschaft ${ }^{47}$ hin zu einer klar hierarchisch untergliederten Gesellschaftsform mit differenzierten sozialen Ebenen erfolgt sei. Diese sollen sich innerhalb der im Zuge der Bestattungspraktik niedergelegten Grabbeigaben ablesen lassen. ${ }^{48}$ Bestärkt wird diese Annahme zudem durch den Nachweis einer aufkommenden monumentalen Architektur ${ }^{49}$ und befestiger Siedlungen, sowie mit der Verwendung des zypro-minoischen Schriftsystems, einem wichtigen Indiz für eine komplexe Gesellschaftsstruktur. Dieser gesellschaftliche Wandel wird als das Resultat einer Elitenbildung gesehen, die sich durch die Akkumulation von Agrarprodukten und verschiedenen Handelsgütern herausgebildet habe, auch wenn eindeutige Beweise für eine zentralisierte Administration der Gesellschaft auf der Insel fehlen. ${ }^{50}$ Ausgelöst worden sei dieser Wandel durch immigrierende Bevölkerungsgruppen oder zumindest deren Imitation durch bereits bestehende lokale Eliten. ${ }^{51}$ Folglich liegen vor allem die Auswirkungen des Kupferhandels und die intensiven Kontakte mit den umgebenden „Hochkulturen“ und den levantinischen Stadtstaaten, allen voran Ugarit, im Blickpunkt der Forschung. ${ }^{52}$ Bereits Steel kritisiert, dass sich die bisherige Zypernforschung dabei besonders für die Spätbronzezeit tendenziell kultur-evolutionistischer Erklärungsmuster bedient habe, um diese Austauschmechanismen mit Rekurs auf ökonomische Bestrebungen und organisierten Handel zu erklären. Alternative Deutungsversuche anhand anthropologischer oder „moderner Methoden“ im Sinne der einleitend diskutierten „Kultur“-Frage

46 Stos-Gale \& Gale (2010); Knapp (2013a) 345, 349.

47 Knapp (2013a) 348.

48 Keswani (2004) 158-159.

49 Fisher (2014).

50 Für alternative Sichtweisen siehe Knapp (2013a) 432-447.

51 Steel (2004a) 143. Wie diese Form der Imitation abgelaufen sein mag, ist aber unklar und hängt mit der kulturellen Einordnung des Materials zusammen. Hier wird deutlich, wie stark das Problem einer Unterscheidung einer archäologischen von einer ethnologischen Kultur im archäologischen Denken verhaftet ist. Dabei ist diese Form der Interaktion eng mit Überlegungen zu formulierten Kontaktzonen verknüpft. Siehe für entsprechende Theorien zu archäologisch fassbarer Immigration, kulturellem Austausch und Kolonisierung beispielsweise Ulf (2009) 93-99 und Burmeister (2000).

52 Steel (2010) 809. 
halten erst seit wenigen Jahren Einzug in die Forschungsmethoden der zyprischen Archäologie. ${ }^{53}$

Am Ende der Bronzezeit zerbricht das weitläufige Austauschsystem des Mittelmeerraumes. Auf Zypern sowie in vielen bedeutenden Siedlungen von Griechenland bis nach Syrien und Anatolien sind extreme kulturelle Umbrüche zu fassen. Während so beispielweise für Griechenland mit dem Späthelladikum IIIC und der submykenischen Zeit verschiedene Phasen der (schriftlosen) Rekonsolidierung (ca. 1190-1000 v. Chr.) in den so genannten Dark Ages diskutiert werden, ist auf Zypern der Wechsel von der Spätbronzezeit in die zyprischgeometrische Zeit vor allem durch die Ansiedlung griechischstämmiger Zuwanderer geprägt. ${ }^{54}$ Im Zuge der Zerstörungen auf Zypern habe also zwar offenbar eine Umstrukturierung der weitläufigen Austauschs- und Handelsmechanismen stattgefunden, deren Intensität aber sei bestehen geblieben. Denn für diese Zeit kann neben dem archäologischen Material erstmals Sprache durch lokale Schriftquellen als ein Vergleichskriterium hinzugezogen werden. Nachgewiesen sind Griechisch und Eteozyprisch, eine bisher nicht identifizierte Sprache. ${ }^{55}$ Beide wurden in einer eigenen zyprischen Schrift geschrieben, dem Zypro-Minoischen Syllabar. Vom Zypro-Minoischen Syllabar wurde die zyprisch-griechische Silbenschrift abgeleitet, in der auch das Zyprisch-Griechische geschrieben wurde. Erst ab dem 9. Jh. v. Chr. tritt gehäuft auch Phönizisch in phönizischer Schrift auf. Diese Entwicklung beeinflusst maßgeblich die Interpretation „fremder“ und „hybrider“ Formen, da die Gleichsetzung verschiedener Kulturgruppen mit eben diesen Sprachen Usus ist. Dennoch sei bis zum 1. Jahrtausend v. Chr. eine Gesellschaft entstanden, die auf der gesamten Insel eine gemeinsame materielle Kultur teile, ein gefestigter Kulturkomplex mit einer einheitlichen materiellen Kultur:

Cyprus entered the 1st millennium $\mathrm{BC}$ with a society that shared a common culture. [...] We do not observe [...] in the material culture, differences that could underscore a distinction between indigenous people and newcomers [...]. The newcomers made no attempt to establish separate enclaves of their own [...] Had it not been for the use of different scripts employed for at least three different languages, it would have been impossible to identify the island's Iron Age multilingual social structure on the basis of the material culture. ${ }^{56}$

Einer einheitlichen materiellen Kultur stehen also drei verschiedene, klar unterscheidbare Sprachen gegenüber. Selbst „Neuankömmlinge“, gemeint sind

53 Steel (2004a) 11 mit Verweisen.

54 Maran (2008); Knapp (2013a) 451.

55 Vgl. Steele (2012).

56 Iacovou (2012) 62-65. 
die erwähnten Einwanderer aus dem mykenischen Griechenland und spätestens im 9. Jh. v. Chr. auch aus der Levante, sind nicht ausdifferenzierbar. So scheint es, als bieten die archäologisch fassbaren Informationen keine Anhaltspunkte für eine Differenzierung kultureller Gruppierungen, obwohl sich in den Schriftquellen durch die parallele Verwendung verschiedener Sprachen (Eteozyprisch, Griechisch und Phönizisch) womöglich weiterhin die Präsenz benachbarter Kulturen zu erfassen sei. Denn am Ende der Spätbronzezeit wird mit der Einführung der Zypro-syllabischen Schrift, die auf Basis der Zyprominoischen Schrift sowohl Griechisch als auch Eteo-zyprisch wiedergibt, die Sprachenvielfalt auffällig. So existieren ab dem 9. Jh. v. Chr. durch die Ansiedlung von Phöniziern in Kition, die diese selbst als Kolonie von Tyros bezeichneten, sowohl Phönizisch, Griechisch im Zypro-syllabischen Schriftbild und die nicht entzifferte eteo-zyprische Sprache parallel. Alle drei Sprachen sind in dieser Zeit sowohl in Wohnräumen, Kultbereichen, im funerären Kontext sowie administrativen Bereich verortet. ${ }^{57}$ Nach Iacovous Zitat (s. o.) überrascht die parallele Verwendung der drei Sprachen im Gegensatz zu der erwähnten Einheitlichkeit materieller Zeugnisse. Insbesondere das Fehlen so genannter Siedlungs-Enklaven zeige, dass besagte Neuankömmlinge innerhalb der Kultur der umgebenden Küstensiedlungen aufgegangen sein müssen. ${ }^{58}$

Doch trotz des Nachweises einer Mehrsprachlichkeit darf weder das Fehlen differenzierbarer Enklaven ausreichen, um darin eine kulturelle Einheit zu postulieren. Noch darf eine Sprache deckungsgleich mit dem Vorhandensein einer Kulturgruppe geltend gemacht werden. Zum einen stellt eine durch Archäologen definierte, typologische Gemeinsamkeit nicht zwingend auch den Ausdruck einer sich selbst als Gemeinschaft verstehenden Gruppierung dar. Diese könnte erst in einem zusammenhängenden Handlungsprozess, beispielsweise einer Bestattungsgemeinschaft, gegeben sein. Die Beständigkeit dieser kulturellen Gruppe kann dabei fluktuieren und bereits nach dem entsprechenden Handlungskomplex (vorübergehend) ausgesetzt werden.

Zum anderen bietet die Grundlage, auf der sich die Rekonstruktion der zyprischen Prähistorie abspielt, methodisch aus verschiedenen Gründen eine große Angriffsfläche. Um archäologisch eine kontinuierliche Entwicklung nachzuzeichnen, ist eine entsprechend große Anzahl von gut datierten und vergleichbaren Fundkontexten notwendig. Doch existieren beispielsweise für den Beginn der Spätbronzezeit keine Daten aus radiometrischen Datierungen kohlenstoffhaltiger Materialien $\left({ }^{14} \mathrm{C}\right)$, so dass der Übergang in diese Epoche nur anhand typologischer Analysen zu fassen ist. Eine Chronologie aufgrund von

57 Iacovou (2013).

58 Iacovou (2012) 63. 
Typen-Abfolgen ist aber zwangsläufig unsicher, denn es ist sogar üblich, dass verschiedene Stile zeitlich parallel verwendet wurden, ein so genannter Stilpluralismus. Chronologisiert man Daten nur aufgrund ihrer typologischen Merkmale, blendet man die Möglichkeit gleichzeitiger Verwendung der unterschiedlichen Typen aus. Die Chronologie bleibt so rein hypothetisch. Selbst für den weiteren Zeitraum von etwa 700 Jahren zwischen Spätzyprisch I und III sind nur $75{ }^{14} \mathrm{C}$-Datensätze aus 8 Fundorten bekannt, die sich außerdem überwiegend auf den Zeitraum Spätzyprisch IIIA beschränken. ${ }^{59}$ Da sich der Großteil der Aufmerksamkeit aber auf wenige dominante Handelszentren beschränkt, ${ }^{60}$ kann die Rekonstruktion einer übergreifenden zyprischen Kultur eigentlich nicht geleistet werden, und alternative Methoden, wie die punktuelle Analyse kultureller Äußerungen, werden notwendig.

\section{Importe, Exotika und Aneignung auf Zypern}

Die Annahme einer gemeinsamen, spätzyprischen Identität, die sich aufgrund von wirtschaftlichen Entwicklungen erkennen ließe, wird trotz der vorgestellten Einwände seit langer Zeit akzeptiert und erweitert. ${ }^{61}$ Der Kupferhandel wird dabei als wichtiger Auslöser für die Etablierung einer lokalen Elite betrachtet, die als Hauptakteur in der Veränderung der kulturellen Situation auf Zypern agiert haben soll. Durch „Anregung“ von außen, die das spätbronzezeitliche Zypern als Verschmelzung verschiedener kultureller Ideen und Überzeugungen darstellen, sei eine politische Einheit, ein so genannter secondary state entstanden. ${ }^{62}$ Formierungsprozesse sind stets Phasen von Konfrontation und notgedrungen eng mit der Beständigkeit und Legitimation von Machtpositionen und -strukturen verbunden, oder sogar das Resultat von und auch der Ausgangspunkt für Gewalt. Entsprechende Hinweise existieren aber einzig durch die erstmalig nachweisbaren Befestigungsanlagen und Stadtmauern mit dem Übergang in die Spätbronzezeit.

Leider beschränkt sich das Wissen über die Strukturen eines Austauschs weitestgehend auf das archäologische Fund-Material. Nur vereinzelt geben Schriftdokumente indirekte Hinweise auf einen mit Zypern gleichzusetzenden Ort namens Alashiya. ${ }^{63}$ Diese in akkadisch verfassten Dokumente stammen

59 Manning (2014).

60 Steel (2004a) 156.

61 Papasavvas (2009) 101; Webb (1999) 298; Karageorghis \& Michaelides (1996); Knapp (1986).

62 Knapp (2013b).

63 Knapp (1996). 
ausschließlich von außerhalb der Insel, beispielsweise aus Ugarit und Amarna. Die ersten Nennungen Alashiyas sind aus Mari, Babylon und Alalakh bekannt und werden in den letzten Abschnitt der zyprischen Mittelbronzezeit datiert, also das 18. und 17. Jh.v. Chr. ${ }^{64}$ Spätere Nennungen sind vor allem aus der politischen Korrespondenz bekannt. Da die lokale, auf Zypro-Minoisch verfasste Sprache bis heute nicht entziffert ist, ${ }^{65}$ ist daher durch Schriftquellen bisher nicht zu klären, ob von einer politischen Einheit auf Zypern ausgegangen werden darf.

Dennoch stehen die beschränkte Zugänglichkeit des Materials und das Postulat eines gesonderten Stellenwertes der Eliten im Widerspruch zu der Annahme eines intensiven Kontaktes aller Bevölkerungsgruppen mit dem „Fremden“. ${ }^{66}$ Auch wo und wie Interaktion und Austausch stattgefunden haben sollen, wird stets an Fundorten wie den großen Küstensiedlungen Enkomi, Hala Sultan Tekke oder Toumba tou Skourou diskutiert, in denen gehäuft Exotika bzw. Importe belegt sind. ${ }^{67}$ Aufgrund dieser Handelszentren werden Qualität und Ausmaß des kulturellen Austausches auf Zypern stark mit der Nähe zu den levantinischen Stadtstaaten und dem intensiven Handel mit dem ägäischen Raum in Verbindung gebracht. Erkennbar sei dieser anhand der Adaptionen alteritärer Formen, dem massiven Aufkommen levantinischer Keramik im 16. und 15. Jh. v. Chr. und mykenischer Keramik während des 14. Jahrhunderts v. Chr., die jeweils als Hinweis einer Adaption fremder Sitten, beziehungsweise als Indiz einer intensiven mykenischen Präsenz auf Zypern herangezogen werden. ${ }^{68}$ Dabei ist aber dringend zwischen importierten Transportgefäßen einerseits, wie beispielsweise Tell el-Yahudiya Ware aus Ägypten, mykenischen Stirrup-Jars oder kanaanitischen Amphoren zu unterscheiden und andererseits Waren, die eine bewusste Nachahmung von Handlungsweisen im Material erkennen lassen. Dies ist beispielsweise in der Verwendung mykenischer Kratere der Fall, einem großformatigen Mischgefäß für Wein, die auch auf elaborierte Trinksitten, einem verstärkten Wein- statt Bierkonsum oder eine gänzlich abgewandelte Verwendung hinweisen können. Diese Erkenntnis kann exemplarisch am Beispiel des amphoroiden Kraters dargestellt werden, der aufgrund stilistischer Überlegungen als Indiz einer Hybridisierung der zyprischen Kultur diskutiert wurde (Abb. 2). Denn während der Keramiktyp des Kraters im griechischen Festland eigentlich weit verbreitet ist, ist diese spezielle Form charak-

64 Knapp (1996) 5.

65 S. o. und Hirschfeld (2010).

66 Keswani (2004) 79-80, 136-139.

67 Knapp (2013a) 39-40.

68 Sherratt (2015) 75-76; Steel (2004a) 154; Steel (2010) 813; Jung (2011) 187-189. 

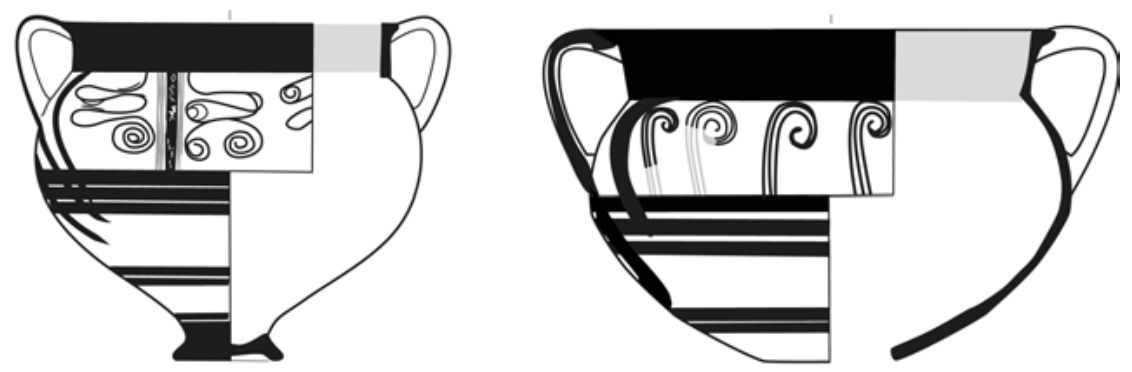

Abb. 2: Amphoroide Kratere aus der Ostägäis (Astypalaia, Armenochori) und Zypern (Palaepaphos Kouklia). Zeichnung von Nora Kuch nach Mountjoy 2009 Fig. 2.1 und 3.1.

teristisch für die Ostägäis und Westanatolien. Der amphoroide Krater aus Kouklia ist jedoch ein Streufund und daher ohne genauen Kontext. Er wurde vermutlich importiert, aber anhand stilistischer Vergleiche und der Glimmeranteile der Keramikmagerung ist erkennbar, dass auch lokal hergestellt Gefäße im mykenischen Stil im Übergang SZ IIIB-C auf IIIC präsent sind und eine Vermischung kretischer, ostägäischer und lokaler Elementen innerhalb der Keramik vorhanden ist. ${ }^{69}$ Diese verhält sich sogar parallel zur Stilentwicklung auf Kreta und auf dem griechischen Festland. ${ }^{70}$ Diese stilistischen Beobachtungen sagen nun allerdings weder etwas über die tatsächliche Verwendung des Kraters aus noch über den Ablauf des Warenaustausches selbst. Immerhin lässt sich sagen: Auch wenn es ohne detaillierte Analysen objektgebundener Kontexte nicht möglich ist, näheres über die Verwendung der einzelnen Gefäße dieses Typus zu erfahren, hat eine selektierte Übernahme stilistischer Eigenarten innerhalb des wahrscheinlich werkstattgebundenen Töpferhandwerks stattgefunden. Wie der spezialisierte Austausch genau organisiert war, ist in Teilen unsicher. Beispielsweise ist unklar, wie stark die Gewichtung zwischen Tribut und durch Eliten oder Einzelpersonen geregelten Handel und deren Auswirkung eingeschätzt werden muss, da die lokale Bevölkerung, wie man allgemein annimmt, nur in kleinformatigen Handel involviert gewesen war. ${ }^{71}$ Eine wichtige Quelle zu diesem Thema liefert die Ladung zweier bronzezeitlicher Handelsschiffe, den Wracks von Galidonya von ca. 1200 v. Chr. und Uluburun von 1300 v. Chr., die jeweils an der Südküste der Türkei gesunken sind. ${ }^{72}$ Transportiert wurden auf dem Schiff von Uluburun zyprisches Kupfer in normierten

69 Siehe ausführlich Mountjoy (2009), bes. 59.

70 Mountjoy (2009) 61.

71 Panagiotopoulos (2012) 54; Steel (2004a) 161.

72 Bass (1967); Yalçin (2005). 
Barren, vor allem so genannten Ochsenhautbarren (siehe entsprechend Abb. 5). Auch wenn die Herkunft der Barrenform vermutlich auf Kreta zu suchen ist und erst für den Zeitraum vor Spätminoisch IB (ca. 1500 v. Chr.) erstmals auch auf Zypern anzunehmen ist, hat sich deren Form in der Forschung insbesondere aufgrund des zyprischen Kupferhandels als ikonisches Element der Handelsmacht der zyprischen Spätbronzezeit herauskristallisiert. ${ }^{73}$ Das Schiff hatte zudem Zinnbarren in einem entsprechenden Verhältnis von 10 : 1 zur Herstellung von Bronze geladen sowie Öl, Elfenbein, Oliven und Granatäpfel, gelagert in levantinischen, mykenischen und zyprischen Gefäßen. Zwar sollen einige persönliche Gegenstände auf eine Mannschaft hindeuten, die aus Ugarit stammte, ein Auslaufhafen des Schiffes kann aber auch nicht daran zweifelsfrei festgemacht werden.

Einen weiteren Beweis für die Quantität exportierter Waren liefern die ugaritischen Schriftquellen und die Amarna-Briefe, in denen Alashiya explizit bezüglich des Gabenaustausches genannt und in denen von großen Mengen an Kupfer und Holz gesprochen wird, das von Thutmosis III angefordert wurde. ${ }^{74}$

Dieser weitreichende Austausch und Handel hatte zweifelsohne Auswirkungen auf die Gesellschaft, wie beispielsweise in den lokalen Bestattungssitten erkennbar ist. Bestattungen waren bereits während der frühen Phasen der Bronzezeit eine wichtige Bühne, um „soziales Kapital“75 zu steigern. Über Generationen hinweg genutzte Kammergräber wurden aufwendig in den Felsen eingearbeitet und mit Bronzen, zahlreichen Gefäßtypen und Modellen ausgestattet. Anscheinend hochrangige Bestattungen wurden zum Teil sogar mit sehr seltenen, exotischen Beigaben versehen. ${ }^{76}$ Insbesondere die Geschirrzusammenstellung deutet dabei auf intensive gemeinschaftliche Aktivitäten und Festlichkeiten hin, in deren Rahmen von identitätsstiftender zwischenmenschlicher Interaktion auszugehen ist. ${ }^{77} \mathrm{Da}$ ein sehr langer Zeitraum überbrückt werden muss, ist eine direkte Übertragung dieser Interpretationen der Grabanlagen in die späte Bronzezeit zwar methodisch fragwürdig, jedoch ähneln sich die Gräber durchaus, und prinzipiell ist von ähnlich gestalteten Grabanlagen auszugehen: in den Felsen eingeschnittene Einzel- oder Mehrkammergräber, die über einen längeren Zeitraum genutzt wurden, so dass Einzelbestattungen sowie Sekundärbestattungen vorliegen. Dabei steht nun aber weniger die Ge-

73 Zur Herkunft der Barrenform siehe Stos-Gale (2011), besonders 226.

74 Steel (2010) 812; Merrillees (2011) 258-259; Raptou (1996); Ockinga (1996) 42, Text 67; Peltenburg (2012) 8-9.

75 Bourdieu (1983).

76 Keswani (2004) 85; Mina (2013).

77 Steel (2010) 811; Keswani (2004) 82, 158-160; Collard (2013); Webb \& Frankel (2008). 
meinschaft, wie es zuvor der Fall war, im Fokus, sondern es kann von einer wachsenden Bezugnahme auf die soziale Stellung der bestatteten und bestattenden Individuen und einer Reflektion einer sozialen Hierarchie während der Spätbronzezeit ausgegangen werden.$^{78}$ Ein wichtiges Fallbeispiel hierfür stellen die Bestattungen bei Enkomi dar, in denen eine Verlagerung der Bestattungsplätze in einen intramuralen Bereich festzustellen ist. ${ }^{79}$ In Verbindung mit der Reduzierung der Personen, die innerhalb einer einzelnen Kammer niedergelegt werden, wird vermutet, dass das Individuum stärker in das Zentrum der Aufmerksamkeit rückt. Wahrscheinlich wird dieser Wandel von einer Differenzierung des sozialen Status begleitet, ${ }^{80}$ der durch den Zugang zu Importgefäßen und Exotika, etwa aus importierten Rohstoffen wie Straußenei, Elfenbein oder Edelmetallen hergestellten Objekten, betont wird.

In der Siedlung Enkomi selbst ist ein Anstieg sogenannter Exotika im Laufe der Spätbronzezeit eindeutig nachzuweisen, bei denen es sich überwiegend um Produkte aus importierten Materialien handelt. ${ }^{81}$ Eine ähnliche Situation ist auch in Kalavasos Ayios Dhimitrios gegeben, einem Gebäudekomplex, der mit großen Lagerkapazitäten ausgestattet war. ${ }^{82}$ In beiden Fällen liefern die Verteilungsmuster der hochwertigen Objekte Hinweise darauf, den entsprechenden Gebäuden eine grundlegend administrative Funktion zuzusprechen. Die Identifizierung einer zugehörigen Elite basiert jedoch ausschließlich auf den reich bestückten Bestattungen; bisher repräsentieren keine Gebäude oder Einrichtungen auf Zypern zweifelsfrei eine stark hierarchisierte Gesellschaft, ${ }^{83}$ und erst in der Eisenzeit sind lokale Kleinkönigreiche sicher nachgewiesen. In der Spätbronzezeit sind aber einzelne Charakteristiken wie Monumentalität, hohe Lagerkapazitäten und der Nachweis einer Schrift relevant, das würde minoischen, mykenischen oder altorientalischen Vergleichsmöglichkeiten entsprechen. Doch trotz der intensiven Kontakte mit Kulturen, die eine ausgeprägte herrschaftliche Selbstdarstellung in Form von Palastanlagen pflegten, konnte bislang weder ein vergleichbarer Gebäudetyp oder ein sonstiger Hinweis auf eine zentrale Administration auf Zypern identifiziert werden.

Weitere monumentale Einrichtungen stellen Heiligtümer dar, die auf $\mathrm{Zy}$ pern erst während der Spätbronzezeit als zweifelsfrei nachgewiesen gelten. Durch stilistische Vergleiche der Sakralarchitektur und ihrem Inventar mit nah-

78 Keswani (2004) 109, 159-160.

79 Steel (2010) 811; Keswani (2004) 86-87.

80 Lorentz (2005) 44.

81 Keswani (2004) 130 Fig. 5.8.

82 South (2008).

83 Knapp (2013a) 445. 

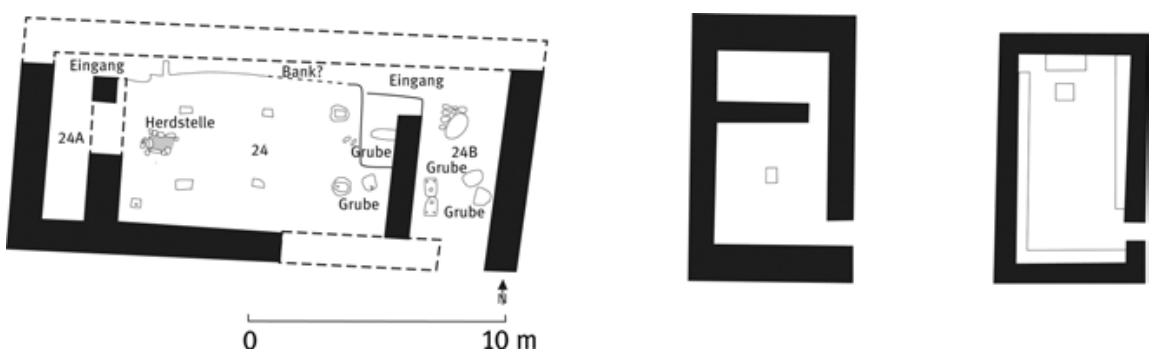

Abb. 3: „Tempel 2“ aus Kition, Area II während Floor IV (SZ IIC), der Knickachstempel „Tempel BA“ aus Tell Mozan (24,5 × $15 \mathrm{~m})$, Frühbronzezeit IVA - Mittelbronzezeit und ein Knickachstempel mit Bankkonstruktionen aus Tell Huera $(10,8 \times 6,5 \mathrm{~m})$ Siedlungsperiode IIA (Spätbronzezeit). Zeichnung Nora Kuch nach Webb 1999 39, fig. 10 und Werner 1994, 126, 127).

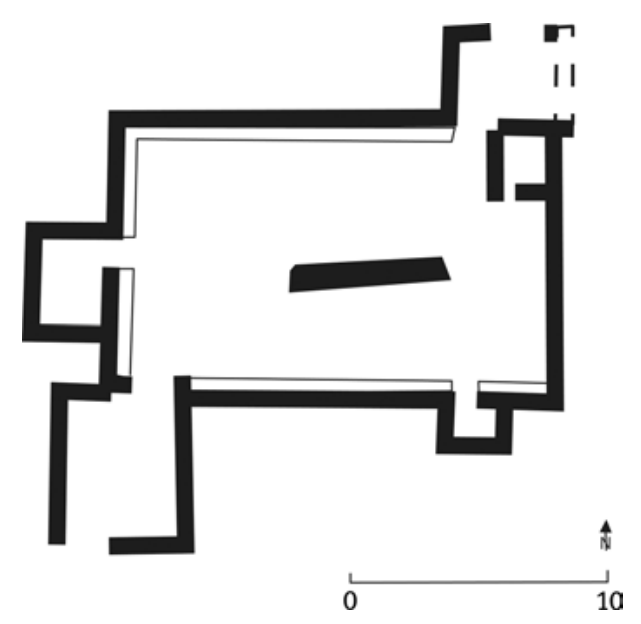

Abb. 4: Umzeichnung des rekonstruierten Heiligtums des Ingot God aus Enkomi, Sol III. Eine freistehende Trennwand separiert den Hauptraum, der mit Steinbänken gesäumt ist. Zeichnung Nora Kuch nach Webb 2001, 73, Fig. 4.

östlichen Paraphernalien wird eine Vermischung aus verschiedenen kulturellen Ansätzen herausgelesen. ${ }^{84}$ Erkennbar sei beispielsweise die Form des Knickachstempels, eines vor allem in Nordmesopotamien genutzten Gebäudetyps, der eine Verschmelzung des Langraum- und Breitraumtempels darstellt (Abb. 3). In einem langrechteckigen Raum befindet sich der Zugang an der Langseite, wobei immer eine $90^{\circ}$ Drehung notwendig ist, um sich der an der Schmalseite aufgestellten Kultstelle zuwenden zu können. ${ }^{85}$ Das so genannte Heiligtum des Ingot God in Enkomi (Abb. 4) und Tempel 2 in Kition (Abb. 3)

84 Webb (1999) 161-162.

85 Werner (1994) 16. 


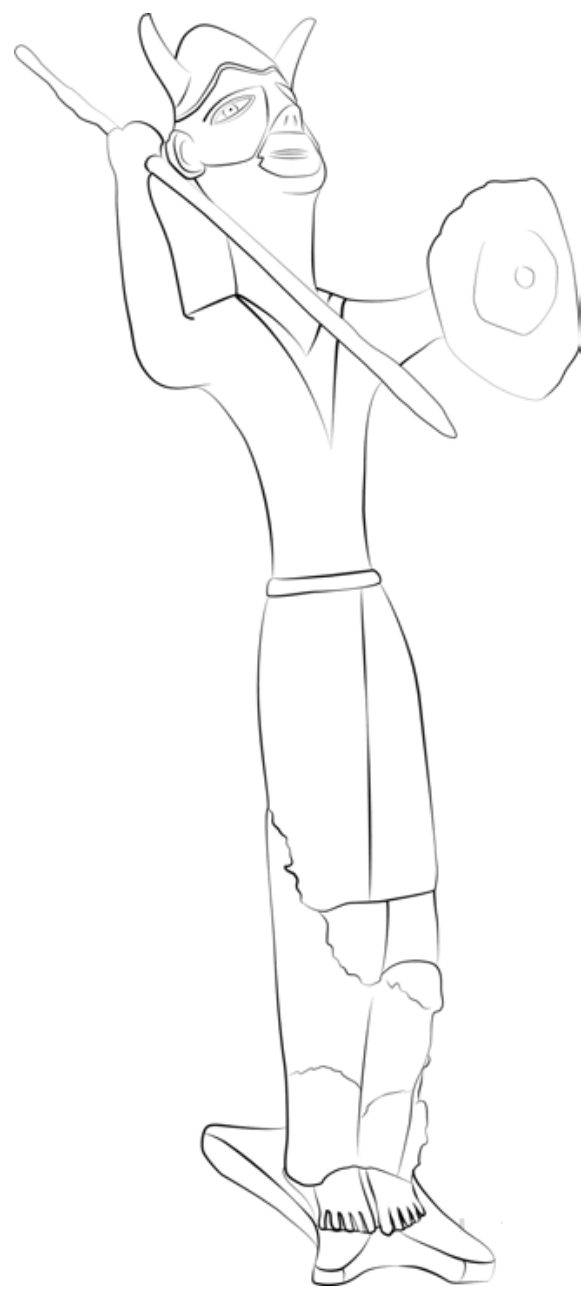

Abb. 5: Umzeichnung des so genannten Ingot God aus Enkomi, Heiligtum des Ingot God. Höhe: 35 cm, SZ IIIA (12001100 v. Chr.) French Excavations. Cyprus Museum, Nikosia. Inv. No. 16.15. Zeichnung Nora Kuch.

sind auf ähnliche Weise aufgebaut. Zudem ist die Präsenz figürlicher Darstellungen mit Anleihen aus dem mykenischen Stilrepertoire bemerkenswert, ${ }^{86}$ aber vor allem liefern Metallfigurinen Raum zu Spekulationen. In diesem Kontext sind vor allem zwei Figurinen, die auf Kupferbarren stehen, in den Blickpunkt geraten. Der so genannte Ingot God (Abb. 5), eine gegossene Kupferfigur $^{87}$ aus dem bereits erwähnten Heiligtum des Ingot God in Enkomi, wird als

86 Knapp (2013a) 395.

87 Nicht Bronze, persönliche Mitteilung von George Papasavvas an Bernard Knapp, Knapp (2012) 39. 
eine der offensichtlichsten Verbindungen von Metallurgie, Kupferverarbeitung, Handwerk und Handel im Rahmen einer übernatürlichen Komponente herangezogen, die von „außen“ inspiriert worden sei. ${ }^{88}$

Die Figur selbst wurde in dem $2 \times 2 \mathrm{~m}$ großen Nordostadyton des Gebäudes gefunden, das innerhalb eines größeren, $11 \times 18 \mathrm{~m}$ umfassenden Hauptraumes situiert ist. Die Figur wurde intentionell niedergelegt und der Raum anschließend unzugänglich gemacht, obwohl das Gebäude womöglich bis zur Aufgabe der Siedlung weiterverwendet wurde. Zudem befanden sich unzählige Weihgaben, Opfergefäße und über 100 Rinderschädel auf dem Boden und den Bänken des Hauptraumes verteilt. Die Figur selbst ist etwa $35 \mathrm{~cm}$ groß und männlich. Sie steht in leicht nach hinten gebeugter, aber aufrechter, Position auf einer Plattform in Form eines Ochsenhautbarrens, der mit einem Befestigungszapfen versehen ist. Die Barrenplattform ist jedoch keine notwendige Stabilisierung, da eine weitere Halterung am Rücken der Figur angebracht war, die allerdings abgebrochen ist.

Die Deutung der Figur als Gottheit beruht weitestgehend auf der Anbringung des Barrens, der als identifizierendes Attribut zu bewerten sei. ${ }^{89}$ Die Figur trägt einen konisch zulaufenden Hut mit Hörnern und einen Vollbart. Da der Nabel verdeckt ist, trägt die Figur womöglich eine Oberkörperbekleidung mit spitz zulaufendem Ausschnitt, es existiert aber weder an den Oberarmen noch an den Handgelenken ein abgrenzender Saum der Bekleidung. Die Figur trägt einen Kilt, der bis zu den Knien reicht, die Beine sind starr und liegen eng aneinander. Der rechte Arm ist nach oben hin angewinkelt und führt einen Speer, der linke Arm ist verkürzt und geht direkt in einen runden Schild über. Ihre Konturen sind prinzipiell sehr exakt und scharf voneinander abgegrenzt, zwar sind sie nicht naturalistisch herausgearbeitet, aber dennoch in ihrer stilisierten Form sehr gleichmäßig und hochwertig. Daher stehen einige handwerklich grob ausgeführte Veränderungen an der Figur im starkem Kontrast. ${ }^{90}$ Anhand detaillierter Analysen, deren Ergebnisse zum Teil noch publiziert werden müssen, ${ }^{91}$ wurde aufgrund des verkürzten Armes und der handwerklichen Mängel bei der Anbringung des Schildes die Möglichkeit späterer Ergänzungen attestiert. Zudem sind grobe Reparaturmerkmale an der unteren Hälfte der Figur auffällig. Wäre dem ursprünglichen Bronzeschmied bei der Herstellung der Figur ein Fehler unterlaufen, wäre die Reparatur entsprechend der Kunstfertigkeit des Schmieds mit größerer Sorgfalt durchgeführt worden. Stattdessen ist

88 Knapp (1986); Kassianidou (2005); Spigelman (2012).

89 Papasavvas (2011) 63.

90 Papasavvas (2011) 59-61.

91 Papasavvas (2011) 61. 
anzunehmen, dass von einer weniger versierten Person erst im Nachhinein ein grober Schaden behoben werden musste oder die Figur gezielt diesen Veränderungen unterzogen wurde. Untypisch ist zudem die Anbringung der Standplatte, die in diesem Fall die Form eines Ochsenhautbarrens hat. ${ }^{92}$ Der Barren selbst wurde womöglich nicht gezielt für diese Aufgabe angefertigt, sondern stellt eine ungleichmäßig eingearbeitete Barren-Miniatur dar. ${ }^{93}$

Die Umarbeitung an der Beinsektion lenkt die Aufmerksamkeit auf die gebeugte Rückenhaltung. Sie beginnt am Knie und somit exakt an der Position, an der auch die handwerklichen Korrekturen einsetzen. Papasavvas nimmt diese Situation zum Anlass, von einer (intentionellen) Umformung einer Figur zu sprechen, die er anhand der typischen waffenführenden Armhaltung und gehörnten Kopfbedeckung als Smiting God aus der Levante identifiziert. ${ }^{94}$ Ungewöhnlich ist nur die Beinstellung, da ein dem Smiting God typischer Ausfallschritt entfernt werden musste und auch ein möglicherweise dolchführender Arm durch einen eher untypischen Schildarm ersetzt wurde. Mit einer intentionellen Umarbeitung der Fußstellung wäre auf diese Weise eine symbolische Umorientierung der Figur einhergegangen, da bereits die Schrittstellung auf Göttlichkeit oder das Menschsein einer Figur hinweisen kann. ${ }^{95}$

Diese Deutung ist aber zu hinterfragen. Die typisch konische Kopfbedeckung mag zwar für Smiting God Darstellungen üblich sein, die Figur des so genannten Horned God aus Enkomi ${ }^{96}$ trägt sie aber ebenfalls und ist keineswegs mit diesen Figurinen stilistisch zu vergleichen. Zudem wurden Smiting God Figuren im Regelfall mit einer Goldfolie versehen, die bereits bei der Herstellung vorgesehen war, so dass im Rücken der Figuren eine dafür nötige Halterung mit eingearbeitet wurde, ${ }^{97}$ die aber sowohl beim Horned wie Ingot God jeweils fehlt.

Anhand der deutlichen Umarbeitungsspuren ist zu sehen, dass fast alle Attribute wie Bewaffnung und Barren nachträglich angebracht wurden. Ihre Deutung und der Stellenwert in der Forschung basieren überwiegend auf der intensiven Adaption oder Umarbeitung einer "fremden“ Gottheit, des Figurentyps des Smiting God, der beispielsweise in Ugarit stark verbreitet war. Wie stark die Versuchung ist, innerhalb dieses Beispiels die scheinbare zyprischindigene Tendenz zur Hybridität erkennen zu wollen, zeigt sich in früheren

92 Papasavvas (2011) 61.

93 Papasavvas (2011) 61; Giumlia-Mair et al. (2011).

94 Papasavvas (2011) 63.

95 Ziffer et al. (2009).

96 Webb (1999) 227.

97 Maran (2011) 68-69. 
Annahmen, die Reparaturstellen an den Füßen und Beinen der Figur seien als ägäische Gamaschen (oder Beinschutzplatten) zu identifizieren. Doch haben sich auch diese als Fehleinschätzung herausgestellt. Die Figur ist uns nur in ihrer letzten Ausgestaltung und im Kontext einer Niederlegungshandlung überliefert, sie stellt keinen Vorzeigefall für einen Hybridisierungsprozess dar. Es fand keine Verbindung verschiedener Elemente zu einer neuen Entität statt, sondern verschiedene Einzelschritte mit einer Bedeutungsverlagerung sind anzunehmen. Die groben Modifikationen an der Figur und ihre klar definierte Ursprungsform liefern dadurch keine gemeinsame Basis für eine bewusste und gesamtheitliche Zusammenführung verschiedener Ideen. Stattdessen ist jeder Zustand der Figur auch für sich stehend $\mathrm{zu}$ bewerten und wäre vielmehr unter objektbiografischen Gesichtspunkten $\mathrm{zu}$ analysieren. ${ }^{98}$

Neben der, wohlgemerkt sehr seltenen, männlichen Figurine zeigt die Interpretation der weiblichen Darstellung auf einem Bronzebarren den forschungsgeschichtlichen Ansatz einer kosmopolitischen Situation, beziehungsweise die Probleme, die durch deren Komplexität entstehen (Abb. 6). Für diese Statuette, benannt nach der 1971 in das Ashmolean Museum integrierten Sammlung Bomford, ist leider kein Fundkontext bekannt. ${ }^{99}$ Ihre Herkunft aus Zypern gilt nur anhand typologischer Merkmale als belegt. ${ }^{100}$ Die Figur ist 9,9 cm groß und stellt eine nackte Frau mit Zopffrisur dar. Eine lange Halskette reicht bis zum Nabel herab, zudem trägt sie einen eng anliegenden Kragen, vielleicht auch mehrere Halsbänder, ihre Hände sind oberhalb des Bauches gefaltet. Die Figur steht auf einem Podest in Form eines Ochsenhautbarrens, an dem im Gegensatz zum Barren des Ingot God keine Podestzapfen angebracht sind. Die Figur ist leicht beschädigt. Die Arme, ein Zopf und ein Stück der Basis fehlen. Sie wird traditionell als eine Göttin gedeutet, der ein Schutzaspekt angehöre, indem sie Fruchtbarkeit in Form von metallischem Reichtum bringe und daher auch mit Aphrodite zu verbinden sei. ${ }^{101}$ Dass es sich hierbei nicht um ein Unikat handeln muss, lässt sich in zwei weiteren Figuren erkennen, die Kouklia Teratsoudhia- und Bairaktar-Figurinen. Diese Figuren sind nur in Fragmenten erhalten, sind der Bomford-Figur aber zumindest in diesen Teilen extrem ähnlich. Die Kouklia Teratsoudhia stammt aus Grab 104 bei Paleapaphos Teratsoudhia, wird Spätzyprisch IIC bis Spätzyprisch IIIA datiert und ist 9,5 cm groß. ${ }^{102}$ Bei der Bairaktar-Figur handelt es sich um einen Streufund

98 Kopytoff (1986); Hahn (2013).

99 Catling (1971); Knapp (1986) 11.

100 Papasavvas (2009) 94; Catling (1971) 24-29.

101 Catling (1971); Matthäus \& Schumacher-Matthäus (1986) 170; Knapp (1986).

102 Karageorghis (1990) 59-61, pl. XXI; Webb (1999) 232. 


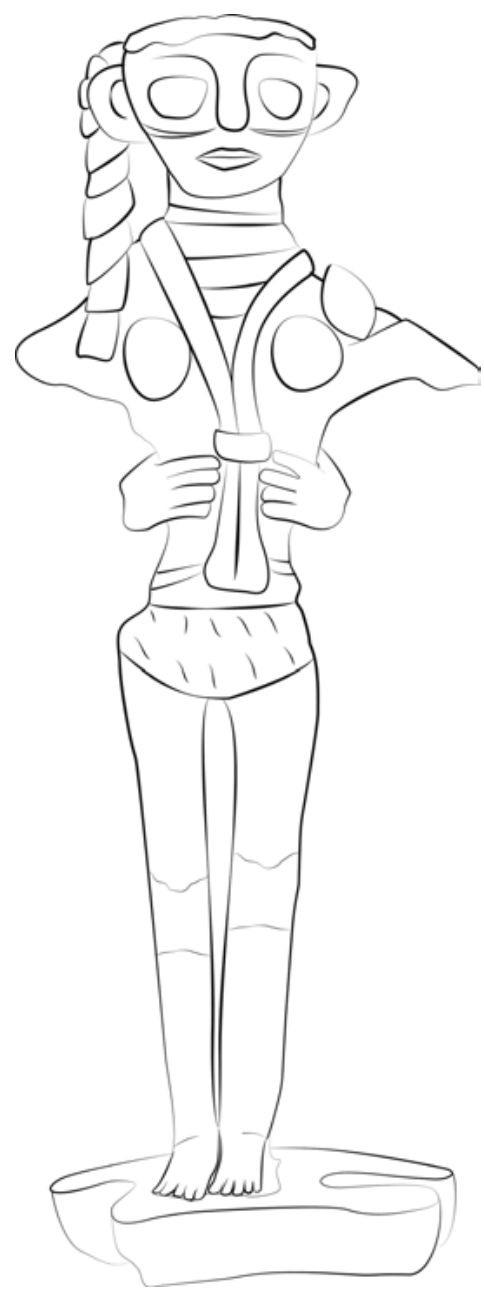

Abb. 6: Umzeichnung der Bomford-Statuette. 12. Jh. v. Chr., Fundort unbekannt. Höhe $9,9 \mathrm{~cm}$. The Ashmolean Museum, Oxford. Zeichnung Nora Kuch.

aus Nikosia. Sie ist zu 10,5 cm erhalten. ${ }^{103}$ Beide stellen ebenfalls eine nackte Frau dar, Schmuck und Haartracht sind identisch zu denen der Bomford-Figur, doch die Ausrichtung der Arme unterscheidet sich. In beiden Fällen sind Teile abgebrochen, und leider fehlen daher auch Beweise für ein mögliches Barrenpodest. Während der Bairaktar-Figur die Füße, eine mögliche Basis und ein Arm fehlen, sind im Fall der Kouklia Teratsoudhia unterhalb der Füße die Reste einer abgebrochenen Basis erkennbar. Wie auch im Fall des Ingot God und der

103 Catling (1971) 20-21, fig. 5; Webb (1999) 232. 
Bomford-Figurine ist die Anbringung einer Basis während dieser Zeitstellung bereits sehr ungewöhnlich, so dass anhand aller bekannten zyprischen Vergleichsfunde bisher nur die Rekonstruktion einer weiteren Barrenfigur ermöglicht wird.

Die Figur als indigen zypriotische Götterfigur ansprechen zu können, sehen Webb und Catling anhand vergleichbarer Figuren als gegeben. Diese Vergleiche sind ohne Barren dargestellt, gemeint ist die so genannte Double Goddess und eine ihr ähnelnde Darstellung auf einem vierseitigen Bronzeständer. Es wird zudem auf die Nichtexistenz von figürlichen Gegenstücken aus Metall im Vorderen Orient verwiesen. ${ }^{104}$ Dennoch ist ein ähnlicher Darstellungstypus u. a. in Terrakotta-Figuren sowohl im Orient als auch in Ägypten weit verbreitet ${ }^{105}$ und führte sogar zu Versuchen, eine Identifizierung der Figuren mit der ägyptischen Gottheit Hathor $\mathrm{zu}$ rechtfertigen. ${ }^{106}$ So weisen die Darstellungen jeweils unterschiedliche Kompositionen auf, beispielsweise auch mit Lotusblüte. Diese Varianz wird traditionell durch einen Kanon von Attributen erklärt. So sei das florale Element als ägyptisierend anzusprechen und zudem, wenn auf einem Löwen stehend, sogar direkt mit Quadesh in Verbindung zu bringen; das Fehlen des Löwen sei Indikator für die Göttin Astarte. ${ }^{107}$ Die Haartracht wird hingegen als der Hathor zugehörig bezeichnet und der Barren sei als indigen zyprisches Attribut zu bewerten. Damit besteht das Problem, dass nicht erkennbar ist, welche Gottheit durch Attribute dargestellt werden sollte, falls überhaupt eine bestimmte göttliche Assoziation damit erzielt werden sollte. Doch durch das Bewusstsein des Auftretens der Aphrodite in der klassischen Antike und ihrer mythologischen Heimat auf Zypern wird in der zyprischen Archäologie der Ansatz einer Genese dieser Gottheit aus all diesen Elementen bestärkt. Für die Bewertung und Interpretation von Figurinen spielt damit eine entscheidende Rolle, dass eine übergeordnete Kategorisierung anhand des Zuschreibens von Attributen bereits zur Annahme einer Verbindung verschiedener kultureller Charakteristika geführt hat, auch wenn nicht einmal klar ist, ob nur eine Frau als Fruchtbarkeitssymbol dargestellt wird oder eine Gottheit.

An dieser Stelle wird nun der dritte Punkt nach Wimmer (s. o. § 1) archäologisch fassbar: die kulturellen Praktiken, die Abgrenzungserscheinungen in sozialen Gruppen markieren, innerhalb derer ein Aushandlungsprozess stattfindet. ${ }^{108}$ Dieser Punkt ist mit dem Ansatz von Punkt zwei (s. u.), der allge-

104 Webb (1999) 232-234 mit Verweis auf Catling (1971) 23-29.

105 Ziffer et al. (2009); Webb (1999) 234.

106 Dazu ausführlich Carbillet (2012).

107 Ziffer et al. (2009) 3.

108 Wimmer (1996) 407. 
meinen Vorstellungen zur Beschaffenheit der Welt verbunden, betont dabei aber eine bewusste „soziale Schließung“ und kulturelle Trennung von Alterität. Ablesbar ist somit eine ablehnende Haltung, die eine klar definierte Selbstwahrnehmung bezeugt. Durch eine starke Differenzierung wäre damit auch archäologisch erkennbar, dass mittels Distinktionspraxis ein Kernelement des Wir-Gefühls einer Gruppe konstruiert wird und sie sich somit im Extremfall als eine historische Abstammungsgemeinschaft verstehen kann, also als Menschen gleicher Kultur und Herkunft. ${ }^{109}$ Ein solcher Abgrenzungsvorgang ist im Beispiel des Ingot God gegeben. Anstelle einer auf die typologische Zusammensetzung der Einzelelemente beruhenden Analyse, deren Ziel eine Benennung der Figur ist, lässt die Umarbeitung und anschließende intentionelle Deponierung der Figur auf unzählige Facetten des Umgangs mit der figürlichen Darstellung schließen. Durch die groben Umarbeitungen, wie die Anbringung von Schild und Kupferbarren, wird deutlich, welch unterschiedlicher Stellenwert diesem Objekt im Laufe seiner Verwendung entgegengebracht worden sein muss. Das ursprüngliche Konzept bei der Herstellung der Figur wurde von einer Person (oder Gruppe) uminterpretiert, deren handwerkliches Können nicht nur begrenzt war, sondern trotz dieses Defizits Änderungen vornahm oder vornehmen ließ. Dabei war offenbar nicht nur die Anbringung eines Miniaturbarrens notwendig, die bereits eine attributive Funktion darstellt und eine bestimmte Assoziation bewirken sollte, sondern auch, dass die Figur zu Schau gestellt werden sollte, wie der Podestzapfen belegt. Mit der abschließenden Deponierung wurde schließlich von einer (oder dieser) Gruppe aktiv Abstand von dieser Intention genommen und nicht mehr die Zurschaustellung der Figur war von Bedeutung, sondern deren Niederlegung in einem stillgelegten Gebäudeteil, der sich in direkter Verbindung mit einem Heiligtum befand.

Doch anstelle diesen Ansatz weiter zu verfolgen, wurden die Erklärungen $\mathrm{zu}$ den ungenauen Korrekturen der Figur auf einen siedlungshistorischen Dezentralisierungsprozess übertragen. Durch das Aufkommen anderer Gottheiten seien verschiedene Bewegungen $\mathrm{zu}$ fassen, die Götterbildnisse bewusst neuen Überzeugungen und Götterbildern entsprechend anpassen würden. ${ }^{110}$ Es lässt sich dabei aber nicht feststellen, ob sich die Figur vor ihrer Deponierung jemals in diesem Gebäude befunden hat, sei es vor oder nach der Umarbeitung. Daher kann anhand dieses Beispiels alleine nicht erörtert werden, ob nun eine Einzelperson die Umarbeitungen zu verantworten hatte oder eine siedlungsweit aktive Gemeinschaft. Doch der Variantenreichtum, mit dem die Figur stets mit einem anderen Zweck belegt wurde, liefert andere und weitreichendere Einblicke

109 Wimmer 1996) 412-413.

110 Papasavvas (2011) 65 mit Verweis auf Knapp (1986); Webb (2001) 79-80. 


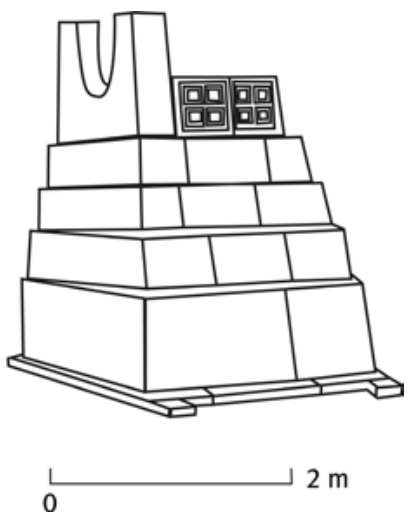

Abb. 7: Rekonstruktionszeichnung eines Altars mit Konsekrationshörnern aus Myrtou Pigadhes, Westhof. Zeichnung Nora Kuch nach Webb 1999, 66.

in die soziokulturellen Prozesse auf Zypern, als die Aneinanderreihung typologischer Elemente, die innerhalb der Figur repräsentiert sind.

Ein letztes auffälliges und konstant auftretendes Element stellt die vielseitige Verwendung von Rind- oder Stierdarstellungen dar. ${ }^{111}$ Das Motiv tritt auf Zypern zahlreich als Applikation, Rhyton, oder als Bukranium auf, letztere beispielsweise in sehr großer Zahl aus Heiligtümern in Enkomi. Herausragend ist zudem eine Situation in Myrthou Pigadhes, einem Heiligtum, das mit Kupferverarbeitung $\mathrm{zu}$ assoziieren ist. ${ }^{12}$ Auf einem Vorhof wird ein großformatiger Altar rekonstruiert (Abb. 7), auf dem sich so genannte Konsekrationshörner befinden, die eindeutig aus dem minoisch-mykenischen Stilrepertoire stammen. ${ }^{113}$ Sie datieren ausnahmslos in Spätzyprisch IIC oder IIIA. Sie wurden bisher im Westhof in Myrtou Pigadhes, Kition Temenos A und B, sowie in Kouklia Palaepaphos innerhalb des Temenos des Sanctuary I gefunden und waren offenbar auf erhöhten Plattformen positioniert. Die Steinhörner sind von unterschiedlicher Größe ( $43 \mathrm{~cm}$ bis 1,20 m) und Herstellungsweise. Die Praxis der dominanten Darstellung von Rindern und deren Interpretation als zentrales Motiv einer Kultur ist aber auch innerhalb der minoischen sowie in der mykenischen Kultur gegeben, unter anderem auch in der Form dieser so genannten Konsekrationshörner. Dennoch unterscheiden sich die zyprischen Hörner von ihren Gegenstücken aus dem ägäischen Raum und dem griechischen Festland. ${ }^{114}$ Zwar sind die Hörner auch auf mehreren importierten Gefäßen mykenischen Typs in Abbildungen nachgewiesen, auf Zypern aber in keinem Fall vor

111 Webb (1999) 57.

112 Webb (1999) 37.

113 Loulloupis (1973).

114 Renfrew (1985) 429; Webb (1999) 176. 
dem 13. Jh. v. Chr. zu fassen. ${ }^{115}$ Nach Webb scheint die direkte Bezugnahme zwischen diesen Hörnern und dem hohen Aufkommen von Stier- oder Rinderdarstellungen unvermeidlich $\mathrm{zu}$ sein. Webb wertet diese sogar als wichtigen Indikator zur bewussten Trennung von sakralen und profanen Räumen. ${ }^{116}$ Während also der Stellenwert dieser Symbolik nicht zu bestreiten ist, stellt die Präsenz dieser Strukturen aber nicht zwingend eine Neuordnung spiritueller Überzeugungen innerhalb der Gesellschaft auf Zypern dar, die eine aus Griechenland emigrierte Bevölkerung ausgelöst haben soll. Sie nimmt im Gegenteil womöglich Bezug auf eine bereits weit in die frühe Bronzezeit zurückgehende Praxis der Integrierung von Rind- und Stiersymbolik innerhalb ritueller Ausdrucksweisen. ${ }^{117}$ So kann sich also als sinnvoll erweisen, die unterschiedlich geartete kulturelle Realisierung dieser Praktiken zu untersuchen, statt die Aufmerksamkeit einzig auf die mykenisch inspirierte Formgebung zu richten.

Hier tritt nun der zweite Punkt nach Wimmer hervor, eine allgemeine, verbindliche Vorstellung über die Beschaffenheit der Welt. Dies betrifft im Prinzip die sozialen Aspekte, wie das Aushandeln von Hierarchien und sozialer Ebenen, in denen sich Individuen in sozialer Interaktion befinden und nach Wimmer einen „sozialen Kompromiss“ aushandeln, den steten Abgleich individueller Normen innerhalb eines gemeinsamen Interaktionsraumes. ${ }^{118}$ Die Ebenen, auf denen diese Interaktionen stattfinden und fassbar sind, können sowohl den alltäglichen Bereich wie die Subsistenzwirtschaft und den Wohnraum betreffen aber auch herausragende Ereignisse, wie Bestattungen oder Festlichkeiten, die sich in vielen Beispielen wie im Heiligtum des Ingot God und MyrtouPigadhes als archäologisch greifbar herausgestellt haben. Die Beigabenmenge innerhalb eines Grabes gilt zwar auch als wichtiger Indikator für den Nachweis einer Hierarchisierung, ist aber nicht als absoluter Indikator zu verstehen. Wie einer bestatteten Person im Kontext einer Bestattung durch die Lebenden eine soziale Position zugesprochen wird, liegt stets innerhalb eines gesellschaftlich konstruierten Rahmens ritueller Handlungskomplexe. Eine auf Herstellungsund Beschaffungsaufwand basierende Wertigkeit, beispielsweise der scheinbar herausragende Wert einer Bestattung mit Gold - ein auf Zypern selten vorkommender Rohstoff - lässt für sich gesehen keine feinjustierte Identifikation sozialer Schichten zu. Die Auswahl der niedergelegten Beigaben ist in erster Linie einem erstaunlich flexiblen Regelwerk unterworfen, materieller Wert definiert nicht zugleich entgegengebrachten Respekt, Ehrerbietung oder andere Qualitä-

115 Webb (1999) 176.

116 Webb (1999) 179.

117 Swiny (2008).

118 Wimmer (1996) 408-412. 
ten im Kontext einer sozialen Gemeinschaft der Bestattenden. Es bezeugt nur die Verfügbarkeit des Materials für die entsprechende Bestattungsgemeinschaft. Doch die Zurschaustellung importierter Beigaben liefert dabei wichtige Hinweise auf die Akzeptanz dieser Objekte innerhalb eines sehr intimen Moments zwischenmenschlicher Interaktion. Innerhalb dieser Plattformen der Interaktion, des Austausches und der Kommunikation liefern das archäologische Material Einblicke auf greifbare Situationen, in denen Veränderung stattfinden kann, Normen und Handlungsweisen zur Disposition stehen und diskutiert werden können und sich auch Adaptionsmechanismen ereignen, seien es neue Trinksitten oder eine scheinbare „Zweckentfremdung“. Dass sich deren handwerkliche Realisierung wie im Beispiel der Konsekrationshörner, des Kraters oder der figürlichen Darstellungen anhand der möglichen Interaktionsmechanismen innerhalb der Gesellschaft vor allem typologisch niederschlägt, ist wenig überraschend und qualitativ nicht viel anders zu bewerten als der allgegenwärtige Prozess des kulturellen Kompromisses. Würde stattdessen von einer vollständigen kulturellen Überprägung des Handelns und Denkens ausgegangen werden, kann die Kultur selbst keine wirkliche Eigendynamik besitzen, sie könnte sich dann nur durch den Kontakt mit anderen Kulturen verändern. ${ }^{119}$

\section{Schlussfolgerungen}

Durch die geostrategische Lage im östlichen Mittelmeerraum wird der Aspekt der Verbindung der Insel mit den umgebenden Kulturkomplexen immer eines der Hauptthemen bleiben, wenn man sich mit der zyprischen Archäologie auseinandersetzt. Die Suche nach Hinweisen für einen „Einfluss von außen“ hat sich im Fall Zypern dabei zu einem methodischen Vorgang entwickelt. Dieser beginnt bei typologischen Charakteristiken von Gefäßtypen, betrifft Verzierungen oder Siedlungsmuster und auch die Grabarchitektur. ${ }^{120}$ Man hat darin Hinweise auf eine „Vermengung unterscheidbarer Kulturen“ erkannt und die Fundstücke folglich als Indikatoren für eine kulturelle Hybridisierung verstanden. ${ }^{121}$ Dieser Prozess wird so nicht nur mit kultureller Identität in Verbindung gebracht, sondern suggeriert, dass Wandel in Technologie, Stil und Material bewusst durchgeführt wurden, um eine Veränderung oder Verbesserung zu erreichen, die sich - s. o. in $\S 3$ das Zitat aus Iacovou (2012) - sogar kontextuell

119 Wimmer (1996) 405.

120 Matthäus (2000).

121 Knapp (2013a) 268. 
auf eine ganze zyprische Kultur übertragen ließe. So wird offenkundig auch das Argument gestärkt, dass anhand von Phasen der Isolation und deren starker Beeinflussung durch „fremde“ Kulturen ein derartiger Wandel fassbar sei. ${ }^{122}$ So können nun verschiedene Schlüsse aus den dargelegten Aussagen gezogen werden, die aber alle zur gleichen Frage führen:

Ist die Spätbronzezeit Zyperns als kulturelles Konglomerat, als eine Form von kulturellem Hybrid zu bewerten?

Die Antwort auf diese Frage ist mehrschichtig. Aufgrund der ungewöhnlich engen Bindungen der Insel zu umgebenden Regionen ist ein Phänomen erkannt, das auch eines der größten Probleme der zyprischen Archäologie im Allgemeinen versinnbildlicht: Die vorherrschende Erwartungshaltung, diese Verbindung auch im Fundmaterial sehen zu können und sie daher als ein definierendes Element einer zyprischen Kultur zu verstehen. Das Fehlen dieses Elements wird im Umkehrschluss als Anzeichen einer Isolationsphase der Insel gewertet, wie sie daher für viele Abschnitte in der zyprischen Prähistorie postuliert wird.

Aneignungsprozesse werden aber auf Zypern überbetont und als kulturelle Besonderheit bewertet. Hintergrund ist, dass zyprische Elemente in den Kontaktregionen für viele Kulturperioden fehlen, die Kontaktverbindungen also nicht reziprok scheinen. In den Augen der Zypernforschung schwebt Zypern damit scheinbar als Sonderform irgendwo zwischen Akkulturation und (postkolonialer) Hybridisierung.

Erst mit der Spätbronzezeit werde das konkreter. ${ }^{123}$ Natürlich ist während der Spätbronzezeit eine Akzentuierung bestimmter Einflüsse wie vorgestellt klar erkennbar. Während beispielsweise die Adaption neuer Trinksitten ${ }^{124}$ oder ikonographischer Elemente als Indiz einer kulturellen Veränderung auf Zypern herangezogen werden, erscheinen Ritualhandlungen hingegen „einheitlich“: Bedeutung von Rind und Metall seien im zyprischen Kulturverständnis weiterverfolgt, aber durch mykenische ${ }^{125}$ oder ugaritische Elemente erweitert worden. In der Forschung diskutiert man damit alltägliche und sakrale Elemente unterschiedlich - ein weiteres Problem der Kategorisierung. Durch die Differenzierung einer Sphäre des Profanen im Kontrast zu einer sakralen Sphäre der Existenz wird so eine Dichotomie zwischen alltäglicher Wahrnehmung und

122 Sherratt (2015) 75.

123 Höflmayer (2011).

124 Steel (2004b) 294-295.

125 Steel (2004) 177-178. 
Übernatürlichem geschaffen. ${ }^{126}$ Diese Art der vereinfachten Differenzierung bei der Kategorisierung antiker Kulturen wurde bereits auf ironische Weise als Ergebnis „Wissenschaftlicher Bequemlichkeit“ betitelt, ${ }^{127}$ spiegelt sich aber weiterhin in den vorgestellten Beispielen wider. Denn anstatt diese Abläufe kontextuell einzubetten, wertet man sie stets als Momente einer gesamtkulturellen Entwicklung aus.

Sobald man dabei Momente übergreifender Einheitlichkeit vermisst, produziert man unbewusst die Idee eines kulturellen Konglomerats, eines Aneinanderklebens kultureller Elemente von außen, nicht einer kulturellen Verarbeitung. Dies ist weder im Sinne der Akkulturation noch der Hybridisierung. Der Situation wird die Eigenschaft, Kultur zu sein, damit einerseits zu-, aber andererseits auch abgesprochen. Der Begriff „Konglomerat“ lädt hier das Thema - bewusst - negativ auf. Denn Abgrenzung gegen andere Kulturen mag durchaus auftreten, ist aber kein definierendes Merkmal von „Kultur“. Wenn Kulturen, sowohl im archäologischen als auch ethnologischen Sinne, nicht unter der Prämisse bewertet werden, dass immer Einflüsse von außen aufgenommen wie auch nach außen weitergegeben werden, entwickelt sich die Methode der Ausdifferenzierung typologischer Merkmale zu einem argumentativen Bumerang. Statt die kulturimmanenten Momente der Aneignung, des Adaptierens, des Weiterkonstruieren, Tradierens (usw.) als Handlung wahrzunehmen, suggeriert das typologische Charakteristikum, diese dahinterstehenden handlungstheoretischen Konzepte weitestgehend ausblenden zu können. Das Objekt wird dann rein anhand seiner Materialität definiert und stets (ungewollt) einer bestimmten „Kultur“ zugeordnet, von der automatisch angenommen wird, sie müsse über innere Einheitlichkeit und abgrenzende Merkmale gegen außen verfügen. Finden sich diese nicht in ausreichendem Maß, „schließt“ man, dass auch die Handlungsträger dieser Kultur in sich nicht einheitlich und nach außen nur unscharf abgegrenzt gewesen seien - mit anderen Worten: keine „Ethnie“ gebildet haben. Nur einer solchen traut man offenbar zu, kulturelle Leistungen $\mathrm{zu}$ vollbringen.

Damit steht ein Objekt zwar stets als wissenschaftlich definierte Einheit im Fokus, nicht aber dessen Herstellung, Verwendung oder Wirkung - den eigentlichen Indizien für menschliche Aktion und Intention. Ein einheitliches Erscheinungsbild der Objekte spiegelt vor allem die spezialisierte Herstellung eines Objekts und damit die Möglichkeit einer zentralisierten Produktion wieder, die Bedeutung von Andersartigkeit, Importen und Hybridisierungsprozessen wird erst im jeweiligen Kontext aufschlussreich.

126 Collard (2013) 111; Eliade (1990) 11; Handler (2011) 45; Panagiotopoulos (2008) 115, 118. 127 Insoll (2004) 8-9. 
Tatsächlich gibt es seit kurzem erste Tendenzen in der Forschung, die Idee phasenweiser Isolation der Insel weniger stark zu betonen und stattdessen zumindest eine passive Einbindung Zyperns in den umgebenden Kulturraum anzunehmen. Mit dieser Annäherung zu einer stärkeren Verbindung aller archäologisch fassbaren Phasen der zyprischen Bronzezeit werden nun auch die weniger deutlich erkennbaren Ausdrucksformen, die mit stetem Austausch in Verbindung stehen, diskutiert. Es werden nun auch die Plattformen für kulturellen Austausch relevant, um die Veränderungen innerhalb der Gesellschaft besser greifen zu können. Doch noch geht die Diskussion nicht weit über einige wenige dieser Plattformen (bspw. Feasting) als Rahmen der sozialen Wahrnehmung und Zurschaustellung von Status hinaus. ${ }^{128}$ Dabei wird an dieser Neuorientierung deutlich, welche Probleme dieser Ansatz des Einflussgebers tatsächlich darstellt, da eine „passive“ Rolle der Zyprioten ${ }^{129}$ während der Interaktion mit Besuchen von außerhalb eigentlich nicht denkbar ist, da stets von einer reziproken Verbindung auszugehen ist. Tatsache aber ist, dass die Präsenz zyprischer Elemente außerhalb der Insel in der Regel als Folge von einfachem Warentausch erklärt wird. Nur selten werden ausführlich die jeweiligen Adaptionsmechanismen, wie beispielsweise im Fall von zyprisch inspirierten Wandapplikationen aus dem mykenischen Griechenland geschehen, diskutiert. ${ }^{130}$

Das Hauptproblem mag wohl in der Kategorisierung selbst liegen. Wie beispielsweise durch den Sprachwissenschaftler Saussure bereits festgestellt wurde, entspricht kein Wort exakt einem anderen:

Aucun mot ne correspond exactement à l'une des notions précisées plus haut; c'est pourquoi toute définition faite à propos d'un mot est vaine; c'est une mauvaise méthode que de partir des mots pour définir les choses ${ }^{131}$

Im Prinzip ist damit jede Definition eines Objektes schlussendlich irreführend, wenn deren Ziel den Versuch darstellt, unumstößliche Abgrenzungen zu erreichen. Stattdessen muss die archäologische Kategorisierung stets die Möglichkeit einer Kombination oder Neuordnung von Elementen für jeden Kontext und Mikrokontext beinhalten, um dem Problem einer einschränkenden Grenzlegung zu entgehen. ${ }^{132}$

128 Steel (2004b); South (2008); Crewe (2009); Fisher (2014); Crewe (2014) 144-145.

129 Mina (2013) 36.

130 Maran (2004); Rahmstorf (2014).

131 „Kein Wort entspricht genau einem der oben genannten Begriffe; daher sind alle Definition von Worten vergebens; Es ist eine schlechte Methode bei der Definition einer Sache mit Worten zu beginnen.“ Saussure (1995 [1916]) 31.

132 Wittgenstein (1953). 
Innerhalb der Debatte der Aneignung und Hybridisierung stellt sich aus archäologischer Sicht daher immer aufs Neue die Frage, wie Fallbeispiele innerhalb eines mehrstufigen Kulturverständnisses betrachtet werden können. Denn die Bewertung prähistorischer Epochen wird dauerhaft mit dem Problem der Kategorisierung verbunden sein. So hängt die Differenzierung archäologischer Kulturen von den Eigenheiten des erhaltenen Materials und der grundsätzlichen Frage nach einer darin ablesbaren Identität ab. Wie eine kulturelle Gemeinschaft definiert wird, ist völlig abhängig von dem Ansatz, der jeweils verfolgt wird. Dabei ist die so genannte Hybridisierung durch Adaption und Handelskontakt auf Zypern nicht als Ergebnis einer kulturellen Neuorientierung zu sehen, sondern im Sinne Wimmers als „kultureller Kompromiss“, einem andauernden und nicht endenden, vielschichtigen Prozess einer allgegenwärtigen Veränderung, der im archäologischen Material nur unterschiedlich stark ausgeprägt ist. Die Inselsituation Zyperns und die Äußerlichkeiten des importierten Materials machen diese nur ungleich auffälliger.

\section{Literaturverzeichnis}

Ackermann, Andreas (2012): Cultural Hybridity: Between Metaphor and Empiricism: Situating Hybridity. In: Stockhammer (2012), Berlin \& Heidelberg: Springer, 5-23.

Albert, Gert \& Sigmund, Steffen (Hgg.) (2010): Soziologische Theorie kontrovers. Wiesbaden: VS-Verlag für Sozialwissenschaft (Kölner Zeitschrift für Soziologie und Sozialpsychologie, Sonderheft 50).

Antonianidou, Sophia (2003): The Impact of Trade on the Society of Cyprus During the Late Bronze Age. Settlements, Artefacts and Social Change. Unveröffentlichte Ph. D. Dissertation, Universität Edinburgh, Department of Archaeology.

Babbi, Andrea \& Bubenheimer-Erhart, Friederike \& Marín-Aguilera, Beatriz \& Mühl, Simone (Hgg.) (2015): The Mediterranean Mirror. Cultural Contacts in the Mediterranean Sea Between 1200 and 750 B. C. International Post-doc and Young Researcher Conference Heidelberg, 6th-8th October 2012. Mainz: Verlag des Römisch-Germanischen Zentralmuseums.

Badisches Landesmuseum Karlsruhe (Hg.) (2008): Zeit der Helden. Die „dunklen Jahrhunderte "Griechenlands 1200-700 v. Chr. Karlsruhe: Badisches Landesmuseum.

Bass, George Fletcher (1967): Cape Gelidonya: a Bronze Age Shipwreck. Philadelphia: American Philosophical Society.

Betancourt, Philip P. \& Susan C. Ferrence (Hgg.) (2011): Metallurgy: Understanding How, Learning Why. Studies in Honor of James D. Muhly. Pennsylvania: INSTAP Academic Press.

Blakolmer, Fritz \& Reinholdt, Claus \& Weilhartner, Jörg \& Nightingale, Georg (Hgg.) (2011): Österreichische Forschungen zur Ägäischen Bronzezeit 2009: Akten der Tagung vom 6. bis 7. März 2009 am Fachbereich Altertumswissenschaften der Universität Salzburg. Wien: Phoibos. 
Bourdieu, Pierre (1983): Ökonomisches Kapital, kulturelles Kapital, soziales Kapital. Originalbeitrag, Übersetzung von Reinhard Kreckel. In: Kreckel (1983), Göttingen: Schwartz, 183-198.

Bourdieu, Pierre (1990): The Logic of Practise. Translated by Richard Nice Stanford: Stanford University Press.

Bräunlein, Peter J. (2012): Material Turn. In: Georg-August-Universität (Hg.), Dinge des Wissens. Sammlungen, Museen und Gärten der Universität Göttingen. Göttingen: Wallstein, 14-28.

Bricker Reifler, Victoria (1975): Intra-cultural variation. American ethnologist 2 (1), 1-18.

Brosius, Christiane \& Polit, Karin M. (Hgg.) (2011): Ritual, Heritage and Identity. The Politics of Culture and Performance in a Globalised World. New Delhi: Routledge Press.

Burmeister, Stefan (2000): Archaeology and Migration. Approaches to an Archaeological Proof of Migration. Current Anthropology 41 (4), August-October 2000, 539-553.

Carbillet, Aurélie (2012): Hathor, La Grande Déesse et l'Industrie du Cuivre Chypriote. In: Georgiou (2012), Oxford: Oxbow Books, 161-176.

Catling, Hector, William (1971): A Cypriot Bronze Statuette in the Bomford Collection. In: Schaeffer (1971), Paris: Mission archéologique d'Alasia, 15-32.

Childe, Vere Gordon (1929): The Danube in Prehistory. Oxford: Clarendon Press.

Childe, Vere Gordon (1933): Races, Peoples and Cultures in Prehistoric Europe. History, New Series Vol. 18 No. 71, 193-203.

Childe, Vere Gordon (1956): Piecing Together the Past: the Interpretation of Archaeological Data. New York: Frederick A. Praeger.

Childe, Vere Gordon (1963): Social Evolution. London: C. A. Watts.

Cline, Eric H. (Hg.) (2010): The Oxford Handbook of the Bronze Age Aegean (ca. 3000$1000 B C$ ). Oxford: Oxford University Press.

Collard, David (2013): When Ancestors Became Gods. The Transformation of Cypriote Ritual in the Late Bronze Age. In: Vasiliki \& Sanders (2013), Leiden: Sidestone Press, 109-119.

Crewe, Lindy (2009): Feasting with the dead? Tomb 66 at Enkomi. In: Kiely (2009), London: British Museum Press, 27-48.

Crewe, Lindy (2014): Expanding and Shrinking Networks of Interaction: Cyprus c. 2200 BC. In: Meller et al. (2015), Halle/Saale: Landesamt für Archäologie, 131-148.

Deger-Jalkotzy, Sigrid (1994): The post-palacial period of Greece: An Aegean prelude to the 11th century B.C. in Cyprus. In: Karageorghis (1994), Nikosia: Leventis Foundation, 1130.

Dommelen, Peter van \& Michael Rowlands (2012): Material concerns and colonial encounters. In: Maran \& Stockhammer (2012), Oxford: Oxbow Books, 20-31.

Duistermaat, Kim \& Ilona Regulski (Hgg.) (2011): Intercultural Contacts in the Ancient Mediterranean. Leuven: Peeters (Orientalia Lovaniensia Analecta, 202).

Egetmeyer, Markus (2013): From the Cypro-Minoan to the Cypro-Greek Syllabaries: Linguistic Remarks on the Script Reform. In: Steele (2013), Cambridge: Cambridge University Press, 107-131.

Eggert, Manfred K. H. (2013): ,Kultur‘. Zum praktischen Umgang mit einem Theoriekonzept. In: Eggert \& Veit (2013), Münster/New York/München/Berlin: Waxmann, 13-62.

Eggert, Manfred K. H. \& Veit, Ulrich (Hgg.) (2013): Theorie in der Archäologie: Zur jüngeren Diskussion in Deutschland. Münster/New York/München/Berlin: Waxmann (Tübinger Archäologische Taschenbücher, 10).

Eliade, Mircea (1990): Das Heilige und das Profane. Vom Wesen des Religiösen. Frankfurt (am Main): Suhrkamp. 
Fischer, Peter M. (Hg.) (2001): Contributions of the Archaeology and History of the Bronze and Iron Age in the Eastern Mediterranean. Wien: Österreichisches Archäologisches Institut.

Fisher, Kevin Douglas (2014): Rethinking the Late Cypriot Built Environments Households and Communities as Places of Social Transformation. In: Knapp \& van Dommelen (2014), Cambridge: Cambridge University Press, 399-416.

Frey, Otto-Herman \& Roth, Helmut \& Dobiat, Claus (Hgg.) (1986): Gedenkschrift für Gero von Merhart: zum 100. Geburtstag. Marburg: Verlag Marie Leidorf (Marburger Studien zur Vor- und Frühgeschichte, 7).

Fröhlich, Siegfried (Hg.) (2000): Kultur. Ein interdisziplinäres Kolloquium zur Begrifflichkeit. Halle (Saale), 18. bis 21. Februar 1999. Halle (Saale): Landesamt für Archäologie.

Galanakis, Yannis \& Wilkinson, Toby \& Bennet, John (Hgg.) (2014): AӨYPMATA: Critical Essays on the Archaeology of the East Mediterranean in Hounour of E. Susan Sherrat. Oxford: Archaeopress.

Georgiou, Artemis (Hg.) (2012): Cyprus. An Island Culture. Society and Social Relations from the Bronze Age to the Venetian Period. Oxford: Oxbow Books.

Giumlia-Mair, Alessandra \& Kassianidou, Vasiliki \& Papasavvas, George (2011): Miniature Ingots from Cyprus. In: Betancourt \& Ferrence (2011), Pennsylvania: INSTAP Academic Press, 11-20.

Hadjisavvas, Sophokles (Hg.) (2010): Cyprus. Crossroads of Civilizations. Nikosia: The Government of the Republic of Cyprus.

Hahn, Hans Peter (2005): Materielle Kultur. Eine Einführung. Berlin: Reimer.

Handler, Richard (2011): The 'Ritualisation of Ritual' in the Construction of Heritage. In: Brosius \& Polit (2011), New Delhi: Routledge Press, 39-54.

Hirschfeld, Nicolle (2010): Cypro-Minoan. In: Cline (2010), Oxford: The Oxford University Press, 373-384.

Hitchcock, Louise Ann \& Laffineur, Robert \& Crowley, Janice L. (Hgg.) (2008): DAIS The Aegean Feast. Proceedings of the 12th International Aegean Conference, University of Melbourne, Centre for Classics and Archaeology, 25-29 March 2008. Liège: Univ. de Liège. (Aegaeum [Annales d'archéologie égéenne de l'Université de Liège et UT-PASP], 29).

Hodder, Ian (2012): Entangled. An Archaeology of the Relationship Between Humans and Things. Oxford: Wiley-Blackwell.

Hodos, Tamar (2006): Local Responses to Colonization in the Iron Age Mediterranean. London \& New York: Routledge.

Höflmayer, Felix (2011): Egyptian Imitations of Cypriote Base Ring Ware in the Eastern Mediterranean. In: Duistermaat \& Regulski (2011), Leuven: Peeters, 347-361.

lacovou, Maria (2012): Early Iron Age. In: Pilidies \& Papadimitriou (2012), Nikosia: Department of Antiquities, 62-65.

lacovou, Maria (2013): The Cypriot Syllabary as a Royal Signature: The Political Context of the Syllabic Script in the Iron Age. In: Steele (2013), Cambridge: Cambridge University Press, 133-152.

Insoll, Timothy (2004): Archaeology, Ritual, Religion. London, New York: Routledge. Jung, Reinhard (2011): Tafeln in Enkomi vom 13. bis zum 12. Jh. v. u. Z.: Neue Töpfe auf dem Tisch oder neue Gäste am Tisch. In: Blakolmer et al. (2011), Wien: Phoibos, 173-196.

Karageorghis, Vassos (Hg.) (1973): Acts of the International Archaeological Symposium 'The Mycenaeans in the Eastern Mediterranean2019, 27 March-2 April 1972. Nikosia: Department of Antiquities. 
Karageorghis, Vassos (1983): Palaepaphos-Skales. An Iron Age Cemetery in Cyprus.

Konstanz: Universitätsverlag Konstanz (Ausgrabungen in Alt-Paphos auf Cypern, 3).

Karageorghis, Vassos (1990): The End of the Late Bronze Age in Cyprus. Nikosia: Published by Pierides Foundation.

Karageorghis, Vassos (Hg.) (1994): Proceedings of the International Symposium Cyprus in the $11^{\text {th }}$ Century B.C. Organized by the Archaeological Research Unit of the University of Cyprus and The Anastasios G. Leventis Foundation, Nicosia 30-31 October, 1993. Nikosia: Leventis Foundation.

Karageorghis, Vassos \& Michaelides, Dēmētrēs (1996): The Development of the Cypriot Economy from the Prehistoric Period to the Present Day. Nikosia: Lithographica.

Karageorghis, Vassos \& Matthäus, Hartmut \& Rogge, Sabine (Hgg.) (2005): Cyprus: Religion and Society. Proceedings of an International Symposium on Cypriote Archaeology, Erlangen, 23-24 July 2004. Möhnesee-Wamel: Bibliopolis.

Karageorghis, Vassos \& Kouka, Ourania (Hgg.) (2009): Cyprus and the East Aegean. Intercultural Contacts from 3000 to 500 BC. An International Archaeological Symposium held at Pythagoreion, Samos, October $17^{\text {th }}-18^{\text {th }}$ 2008. Nikosia: The A. G. Luventus Foundation.

Kassianidou, Vassos (2005): Was Copper Production Under Divine Protection in Late Bronze Age Cyprus? Some Thoughts on an Old Question. In: Karageorghis et al. (2005), Möhnesee-Wamel: Bibliopolis, 127-142.

Keswani, Priscilla Schuster (2004): Mortuary Ritual and Society in Bronze Age Cyprus. London: Oakville.

Kiely, Thomas Michael (Hg.) (2009): Ancient Cyprus in the British Museum: Essays in honour of Dr. Veronica Tatton-Brown. British Museum Research Publication 180, London: British Museum Press.

Knapp, Arthur Bernard (1986): Copper Production and Divine Protection: Archaeology, Ideology and Social Complexity on Bronze Age Cyprus. Göteborg: Paul Åström.

Knapp, Arthur Bernard (1994): Emergence, Development, and Decline on Bronze Age Cyprus. In: Mathers \& Stoddart (1994), Sheffield: Equinox Publishing, 271-303.

Knapp, Arthur Bernard (Hg.) (1996): Near Eastern and Aegean Texts from the Third to the First Millenia BC. New York: Inst. of Cypriot Studies, Univ. at Albany (Sources for the History of Cyprus, II).

Knapp, Arthur Bernard (2012): Matter of Fact: Transcultural Contacts in the Late Bronze Age Eastern Mediterranean. In: Maran \& Stockhammer (2012), Oxford: Oxbow Books, 32-50.

Knapp, Arthur Bernard (2013a): The Archaeology of Cyprus. From Earliest Prehistory Through the Bronze Age. Cambridge: Cambridge University Press.

Knapp, Arthur Bernard (2013b): Revolution Within Evolution: The Emergence of a 'Secondary State' on Protohistoric Bronze Age Cyprus. Levant. 45(1), 19-44.

Knapp, Arthur Bernard \& Webb, Jennifer M. \& McCarthy, Andrew (Hgg.) (2013): J. R. B. Stewart. An Archaeological Legacy. Uppsala: Åström (Studies in Mediterranean Archaeology 139).

Knapp, Arthur Bernard (Hg.) (2014): The Archaeology of Cyprus. From Earliest Prehistory Through the Bronze Age. Cambridge: Cambridge University Press.

Knapp, Arthur Bernard \& van Dommelen, Peter (Hgg.) (2014): The Cambridge Prehistory of the Bronze and Iron Age Mediterranean. Cambridge: Cambridge University Press.

Kreckel, Reinhard (Hg.) (1983): Soziale Ungleichheiten. Göttingen: Schwartz (Soziale Welt, Sonderband 2). 
Kroeber, Alfred L. \& Kluckhohn, Clyde (1963): Culture: A Critical Review of Concepts and Definitions. New York: Vintage Books.

Lichardus, Jan (Hg.) (1991): Die Kupferzeit als historische Epoche. Bonn: Rudolf Habelt (Saarbrückener Beiträge zur Altertumskunde, 55).

Lichardus, Jan (1991a): Die Kupferzeit als historische Epoche. Eine forschungsgeschichtliche Einleitung. In: Lichardus (1991), Bonn: Rudolf Habelt, 13-32.

Lorentz, Kirsi (2005): Late Bronze Age Burial Practices: Age as a Form of Social Difference. In: Karageorghis et al. (2005), Wöhmesee-Wamel: Bibliopolis, 41-56.

Lo Schiavo, Fulvia \& Muhly, James David \& Maddin, Robert \& Giumlia-Mair, Alessandra (Hgg.) (2009): Oxhide Ingots in the Central Mediterranean. Biblioteca di Antichità Cipriote 8, Rom: A. G. Leventis Foundation.

Loulloupis, M. (1973): Mycenaean ,Horns of Consecration' in Cyprus. In: Karageorghis (1973), Nikosia: Department of Antiquities, 225-244.

Lüning, Jens (1972): Zum Kulturbegriff im Neolithikum. Prähistorische Zeitschrift 47, Berlin: DeGruyter, 145-173.

Maguire, Richard \& Chick, Jane (Hgg.) (2016): Approaching Cyprus: Proceedings of the PostGraduate Conference of Cypriot Archaeology (PoCA) held at the University of East Anglia, Norwich, 1st-3rd November 2013. Cambridge: Cambridge Scholars Press.

Manning, Sturt W. (2014): Appendix: A New Radiocarbon Chronology for Prehistoric and Protohistoric Cyprus, ca. 11,000-1050 Cal BC. In: Knapp (2014), Cambridge: Cambridge University Press, 477-533.

Manning, Sturt W. \& B. Weniger, \& A. K. South \& B. Kling \& P. I. Kuniholm \& James D. Muhly \& Sophocles Hadjisavvas \& D. A. Sewell \& G. Cadogan (2001): Absolute age range of the Late Cypriot IIC period on Cyprus. Antiquity 75, 328-340.

Maran, Joseph (2004): The Spreading of Objects and Ideas in the Late Bronze Age Eastern Mediterranean: Two Case Examples from the Argolid of the 13th and 12th Centuries B.C. Bulletin of the American Schools of Oriental Research 336, 11-30.

Maran, Joseph (2008): Nach dem Ende. Tiryns - Phönix aus der Asche. In: Badisches Landesmuseum Karlsruhe (2008), Karlsruhe: Badisches Landesmuseum, 63-73.

Maran, Joseph (2011): Evidence for Levantine Religious Practice in the Late Bronze Age Sanctuary of Phylakopi on Melos? Eretz-Israel, Archaeological, Historical and Geographical Studies 30, 65-73.

Maran, Joseph (2012): One World Is Not Enough: The Transformative Potential of Intercultural Exchange in Prehistoric Societies. In: Stockhammer (2012), Berlin \& Heidelberg: Springer, 59-66.

Maran, Joseph \& Stockhammer, Philipp Wolfgang (Hgg.) (2012): Materiality and Practice. Transformative Capacities of Intercultural Encounters. Oxford: Oxbow.

Mathers, Clay \& Stoddart, Simon (Hgg.) (1994): Development and Decline in the Mediterranean Bronze Age. Sheffield Archaeological Monographs 8. Sheffield: Equinox Publishing.

Matthäus, Hartmut (2000): Eine kosmopolitische Hochkultur. Zypern von 1600-1100 v. Chr. In: Rogge (2000), München: Waxmann, 91-125.

Matthäus, Hartmut \& Gisela Schumacher-Matthäus (1986): Zyprische Hortfunde. Kult und Metallhandwerk in der späten Bronzezeit. In: Frey et al. (1986), Marburg: Verlag Marie Leidorf, 129-191.

Meller, Harald \& Arz, Helge Wolfgang \& Jung, Reinhard \& Risch, Roberto (Hgg.) (2015): 2200 BC - Ein Klimasturz als Ursache für den Zerfall der Alten Welt? 2200 BC - 
A climatic breakdown as a cause for the collapse of the old world? 7. Mitteldeutscher Archäologentag vom 23. bis 26. Oktober 2014 in Halle (Saale). Halle/Saale.

Merrillees, Robert S. (1992): The absolut chronology of the Bronze Age in Cyprus: a revision. Bulletin of the American Schools of Oriental Research 288, 47-52.

Merrillees, Robert S. (2011): Alashiya: A Scientific Quest for its Location. In: Betancourt \& Ferrence (2011), Pennsylvania: INSTAP Academic Press, 255-267.

Mina, Maria (2013): Exotica in Early and Middle Bronze Age Cyprus: Exploring Aspects of the Secular and Sacred Economy. In: Knapp et al. (2013), Uppsala: Åström, 33-45.

Mountjoy, Penelope A. (2009): Cyprus and the East Aegean: LH IIIC pottery connections. In: Karageorghis \& Kouka (2009), Nikosia: The A.G. Leventis Foundation, 59-71.

Ockinga, Boyo (1996): Hieroglyphic Texts from Egypt. In: Knapp (1996), New York: Institute of Cypriot Studies, Univ. at Albany, 42-50.

Panagiotopoulos, Diamantis (2008): Natur als sakraler Raum in der minoischen Kultur. Archiv für Religionsgeschichte 10, 115-142.

Panagiotopoulos, Diamantis (2012): Encountering the foreign. (De-)constructing alterity in the archaeologies of the Bronze Age Mediterranean. In: Maran \& Stockhammer (2012), Oxford: Oxbow Books, 51-61.

Papasavvas, George (2009): The Iconography of the Oxhide Ingots. In: Lo Schiavo et al. (2009), Rom: The A.G. Leventis Foundation, 83-132.

Papasavvas, George (2011): From Smiting to Smithing. In: Betancourt \& Ferrence (2011), Pennsylvania: INSTAP Academic Press, 59-68.

Pelt, Paul van (Hg.) (2013): Archaeology and Cultural Mixture. Cambridge: Department of Archaeology (Archaeological Review from Cambridge, 28.1).

Peltenburg, Edgar (1996): From Isolation to State Formation in Cyprus, c. 3500-1500 BC. In: Karageorghis \& Michaelides (1996), Nikosia: Lithographica, 17-43.

Peltenburg, Edgar (2012): Text Meets Material in Late Bronze Age Cyprus. In: Georgiou (2012), Oxford: Oxbow Books, 1-23.

Pilidies, Despina \& Papadimitriou, Nikolas (Hgg.) (2012): Ancient Cyprus: Cultures in Dialogue. Exhibition Catalogue. Nikosia: Department of Antiquities.

Rahmstorf, Lorenz (2014): A 'wall-bracket' from Kandia in the Argolid: notes on the local character and function of an 'east Mediterranean' artefact of the Late Bronze Age/Early Iron Age. In: Galanakis et al. (2014), Oxford: Archaeopress, 187-195.

Raptou, Eustathios (1996): Contribution to the Study of the Economy of Ancient Cyprus: Copper - Timber. In: Karageorghis \& Michaelides (1996), Nikosia: Lithographica, 249259.

Renfrew, Colin (1985): The Archaeology of Cult. The Sanctuary at Phylakopi. London: British School of Archaeology at Athens.

Rogge, Sabine (Hg.) (2000): Zypern. Insel im Brennpunkt der Kulturen. München: Waxmann.

Saussure, Ferdinand de (1995 [1916]): Cours de linguistique générale. Publié par Charles Bailly et Albert Séchehaye avec la collaboration de Albert Riedlinger. Édition critique préparée par Tullio de Mauro. Paris: Payot (Payothèque).

Schaeffer, Claude Frédéric-Armand (Hg.) (1971): Alasia I. Paris: Mission archéologique d'Alasia; IV, Paris: (Mission archéologique d'Alasia) (Mission archéologique d'Alasia, IV).

Schreiber, Stefan (2013): Archäologie der Aneignung. Zum Umgang mit Dingen aus kulturfremden Kontexten. Forum Kritische Archäologie 2, 48-123. Online verfügbar unter http://www.kritischearchaeologie.de/ausgaben.php\#fka2 (Stand 20. 05. 2018). 
Sherratt, Susan (2015): Cyprus and the Near East: Cultural Contacts (1200-750 BC). In: Babbi et al. (2015), Mainz: Verlag des Römisch-Germanischen Zentralmuseums, 7184.

Spigelman, Matthew (2012): Copper and Cult in Bronze Age Cyprus. In: Georgiou (2012), Oxford: Oxford Books, 133-152.

South, Alison (2008): Feasting in Cyprus: a View from Kalavasos. In: Hitchcock et al. (2008), Liège: Univ. de Liège, 309-316.

Steel, Louise (2004a): Cyprus Before History. From the Earliest Settlers to the End of the Bronze Age. London: Duckworth.

Steel, Louise (2004b): A Goodly Feast... A Cup of Mellow Wine. Feasting in Bronze Age Cyprus. Hesperia 73, 281-300.

Steel, Louise (2010): Cyprus. In: Cline (2010), Oxford: Oxford University Press, 804-819.

Steele, Philippa M. (2012) Eteocypriot: Linguistic and Archaeological Evidence. In: Georgiou (2012), Oxford: Oxbow Books, 122-132.

Steele, Philippa M. (ed.) (2013): Syllabic Writing on Cyprus and its Context. Cambridge: Cambridge University Press.

Steele, Philippa M. (2013): A Linguistic History of Ancient Cyprus: The Non-Greek Languages and their Relations with Greek, c. 1600-300 B.C. Cambridge: CUP.

Steele, Philippa M. (2016): A Linguistic Approach to Population Movements to Ancient Cyprus. In: Maguire \& Chick (2016), Cambridge: Cambridge Scholars Press, 2-16.

Stevens, Peter A. J. (2016): Ethnicity and Racism in Cyprus. National Pride and Prejudice? Hampshire: Palgrave Macmillan (Mapping Global Racisms).

Stockhammer, Philipp Wolfgang (Hg.) (2012) Conceptualizing Cultural Hybridization: A Transdisciplinary Approach. Papers of the Conference, Heidelberg, 21st-22nd September 2009. Berlin \& Heidelberg: Springer (Transcultural Research. Heidelberg Studies on Asia and Europe in a Global Context).

Stockhammer, Philipp Wolfgang (2013): From Hybridity to Entanglement, from Essentialism to Practise. In: Pelt (2013), Cambridge: Department of Archaeology, 11-28.

Stos-Gale, Zofia Anna \& Noel Gale (2010): Bronze Age metal artefacts found on Cyprus metal from Anatolia and the Western Mediterranean. Trabajos de prehistoria 67 (2), 389-403.

Stos-Gale, Zofia Anna (2011): „Biscuits with Ears”: A Search for the Origin of the Earliest Oxhide Ingots. In: Betancourt \& Ferrence (2011), Pennsylvania: INSTAP Academic Press, 221-230.

Swiny, Stuart (2008): Of Cows, Copper, Corners, and Cult: The Emergence of the Cypriot Bronze Age. Near Eastern Archaeology 71 (1-2), 41-51.

Tringham, Ruth (1983): V. Gordon Childe 25 Years after: His Relevance for the Archaeology of the Eighties. Journal of Field Archaeology 10 (1), 85-100.

Ulf, Christoph (2009): Rethinking Cultural Contacts, Ancient West \& East 8, 81-132.

Vasiliki, G. Koutrafouri \& Sanders, Jeff (Hgg.) (2013): Ritual Failure. Archaeological Perspectives. Leiden: Sidestone Press.

Webb, Jennifer M. (1999): Ritual Architecture, Iconography and Practice in the Late Cypriot Bronze Age, Jonsered: P. Åströms.

Webb, Jennifer M. (2001): The Sanctuary of the Ingot God at Enkomi. A New Reading of its Construction, Use and Abandonment. In: Fischer (2001), Wien: Österreichisch Archäologisches Institut, 69-82.

Webb, Jennifer M. \& David Frankel (2008): Fine ware ceramics, consumption and commensality: mechanisms of horizontal and vertical integration in Early Bronze Age Cyprus. In: Hitchcock et al. (2008), Liège: Univ. de Liège, 287-295. 
Werner, Peter (1994): Die Entwicklung der Sakralarchitektur in Nordsyrien und

Südostkleinasien. Vom Neolithikum bis in das 1. Jt. v. Chr. München/Wien: Profil Verlag (Münchener vorderasiatische Studien, 15).

Wimmer, Andreas (1996): KULTUR. Zur Reformulierung eines sozialanthropologischen

Grundbegriffs. Kölner Zeitschrift für Soziologie und Sozialpsychologie 48 (3), 401-425.

Wimmer, Andreas (2010): Kultur als Kompromiss. In: Albert \& Sigmund (2010), Wiesbaden: VS-Verlag für Sozialwissenschaft, 411-426.

Wittgenstein, Ludwig (1953): Philosophische Untersuchungen. Oxford: Blackwell.

Wotzka, Hans-Peter (1993): Zum traditionellen Kulturbegriff in der prähistorischen Archäologie. In: Paideuma: Mitteilungen zur Kulturkunde 39, 25-44.

Wotzka, Hans-Peter (2000): „Kultur“ in der deutschsprachigen Urgeschichte. In: Fröhlich (2000), Halle (Saale): Landesamt für Archäologie, 55-80.

Wulf, Christoph (2011): Performativity and Dynamics of Intangible Cultural Heritage. In: Brosius \& Polit (2011), New Delhi: Routledge Press, 76-94.

Yalçın, Üsal \& İpek, Önder \& Medenbach,Olaf (Hgg.) (2005): Das Schiff von Uluburun. Welthandel vor 3000 Jahren. Katalog der Ausstellung im Deutschen Bergbau-Museum Bochum vom 15. Juli 2005 bis 16. Juli 2006. Bochum: Deutsches Bergbau-Museum.

Ziffer, Irit \& Bunimovitz, Shlomo \& Lederman, Zivi (2009): Divine or Human? An Intriguing Late Bronze Age Plaque Figurine from Tel Beth-Shemesh. Ägypten und Levante 19, 333-342. 